\section{TRAF6-IRF5 kinetics, TRIF, and biophysical factors drive synergistic innate responses to particle-mediated MPLA-CpG co-presentation}

\author{
P. Pradhan ${ }^{1,2,3 *}$, R. Toy ${ }^{1 *}$, N. Jhita ${ }^{4}$, A. Atalis ${ }^{1}$, B. Pandey ${ }^{1}$, A. Beach ${ }^{1}$, E. L. Blanchard ${ }^{1}$, \\ S. G. Moore ${ }^{2}$, D. A. Gaul ${ }^{2}$, P. J. Santangelo ${ }^{1}$, D. M. Shayakhmetov ${ }^{4,5}$, K. Roy ${ }^{1,2,3 \dagger}$
}

Innate immune responses to pathogens are driven by co-presentation of multiple pathogen-associated molecular patterns (PAMPs). Combinations of PAMPs can trigger synergistic immune responses, but the underlying molecular mechanisms of synergy are poorly understood. Here, we used synthetic particulate carriers co-loaded with monophosphoryl lipid A (MPLA) and CpG as pathogen-like particles (PLPs) to dissect the signaling pathways responsible for dual adjuvant immune responses. PLP-based co-delivery of MPLA and CpG to GM-CSF-driven mouse bone marrow-derived antigen-presenting cells (BM-APCs) elicited synergistic interferon- $\beta$ (IFN- $\beta$ ) and interleukin-12p70 (IL-12p70) responses, which were strongly influenced by the biophysical properties of PLPs. Mechanistically, we found that MyD88 and interferon regulatory factor 5 (IRF5) were necessary for IFN- $\beta$ and IL-12p70 production, while TRIF signaling was required for the synergistic response. Both the kinetics and magnitude of downstream TRAF6 and IRF5 signaling drove the synergy. These results identify the key mechanisms of synergistic Toll-like receptor 4 (TLR4)-TLR9 co-signaling in mouse BM-APCs and underscore the critical role of signaling kinetics and biophysical properties on the integrated response to combination adjuvants.

\section{INTRODUCTION}

Immune adjuvants have been widely used to boost the potency of weakly immunogenic vaccines against infectious diseases and cancers $(1,2)$. Alum-based adjuvants, first licensed in the 1920s, are used clinically to induce broad innate immune responses, but their lack of specificity makes them unsuitable for a broad range of vaccines and raises concerns for long-term tolerability and potential side effects (3). On the other hand, certain molecules from viruses, bacteria, and other parasites-collectively known as pathogen-associated molecular patterns (PAMPs) - engage with pattern recognition receptors (PRRs) in mammalian cells, located on the cell surface, on endosomes, and in the cytoplasm, to trigger highly specific innate immune responses (4).

Highly successful vaccines in human history are composed of live-attenuated or inactivated pathogens, which present multiple adjuvants and antigens assembled on a particulate structure to the immune system and generate protective immunity $(1,5)$. For emerging vaccines consisting of recombinant proteins, peptides, or nucleic acids, various PAMPs are being investigated as adjuvants. The triggering of Toll-like receptors (TLRs) on cell membranes and endosomes, and RIG-I-like receptors and cGAS-STING receptors in the cytoplasm, induces potent immunity (6-9). Although pathogens carry multiple PAMPs and antigens within a unified structure, most vaccine research with PAMPs has historically involved investigations of antigens with independent soluble adjuvants. Recently, particu-

\footnotetext{
${ }^{1}$ The Wallace H. Coulter Department of Biomedical Engineering, Georgia Institute of Technology, Atlanta, GA, USA. ${ }^{2}$ The Parker H. Petit Institute for Bioengineering and Biosciences, Georgia Institute of Technology, Atlanta, GA, USA. ${ }^{3}$ Marcus Center for Therapeutic Cell Characterization and Manufacturing, Georgia Institute of Technology, Atlanta, GA, USA. ${ }^{4}$ Lowance Center of Human Immunology, Department of Pediatrics and Medicine, Emory University School of Medicine, Atlanta, GA, USA. ${ }^{5}$ Emory Vaccine Center, Emory University School of Medicine, Atlanta, GA, USA. *These authors contributed equally to this work.

tCorresponding author. Email: krish.roy@gatech.edu
}

late carriers and combination adjuvants have gained increasing interest to better mimic the composition of pathogens and elicit more effective immune responses (10-13). The main caveat in combination adjuvant delivery is that immune adjuvants have different solubility and diffusion characteristics. When delivered as soluble molecules in vivo, their concurrent presentation and efficient intracellular delivery to innate immune cells are difficult to achieve, especially when one combines a hydrophobic adjuvant [e.g., monophosphoryl lipid A (MPLA)] with a highly hydrophilic adjuvant (e.g., $\mathrm{CpG}$ ). In addition, rapidly diffusible adjuvants (like $\mathrm{CpG}$ ) induce acute systemic toxicity and require a particulate carrier to prevent rapid diffusion, reduce toxicity, and enable targeted delivery to immune cells $(14,15)$. Particle systems solve the challenges of intracellular delivery, reduce systemic toxicity, and facilitate the co-delivery of diverse types of antigens and adjuvants (16-19). By strengthening immune responses, particle systems can safely enhance vaccine efficacy or drive potent antitumor effects (20-22).

While some TLR adjuvants are antagonistic to each other (e.g., TLR3 and TLR7), other TLR adjuvant combinations drive synergistic immune responses (23-25). For instance, nanoparticles with an influenza antigen and co-encapsulated TLR4 and TLR7 adjuvants drive strong immune responses that result in exceptional protection against the flu (26). The combination of adjuvants activating TLR4 and TLR9, both PRRs expressed on Gram-negative bacteria, also drives potent, synergistic immune response (27). Activation of TLR4 on the cell membrane and the endosome drives the production of type I interferons (IFNs) and proinflammatory cytokines through nuclear factor $\kappa \mathrm{B}(\mathrm{NF}-\kappa \mathrm{B})$ activation (28). Similarly, TLR9 activation in the endosome induces proinflammatory cytokine and type I IFN production $(29,30)$. Our group has demonstrated that pathogen-like particles (PLPs) with two clinically relevant TLR adjuvants (MPLA, a TLR4 adjuvant, and CpG, a TLR9 adjuvant) induce synergistic innate immune responses in bone marrowderived antigen-presenting cells (BM-APCs) and adaptive immune 
responses in vivo (31). When the PLPs with adjuvants are delivered along with surface-bound ovalbumin antigen, an enhanced humoral immune response is observed when compared to PLPs with single adjuvants and antigen. One group has postulated that synergy is a result of increased TLR9 recruitment to the endosome after TLR4 activation on the plasma membrane. However, the precise cellular and molecular mechanism enabling TLR4-TLR9 synergy is unknown (32).

Here, we investigated the TLR4 and TLR9 signaling pathways to identify the underlying mechanism driving the synergistic innate immune response to cis presentation of MPLA and CpG in particulate carriers on mouse BM-APCs differentiated with granulocytemacrophage colony-stimulating factor (GM-CSF). We demonstrate that biophysical properties significantly affect the innate immune response-specifically, high CpG density dual adjuvant-loaded particles increase the magnitude of synergistic IFN- $\beta$ and interleukin12 p70 (IL-12p70) responses to MPLA and CpG. We also show that although the cytokine response from the MPLA dose, when used as a single adjuvant, is minimal, the TLR4 signaling arm is necessary for the dual adjuvant synergistic response. Specifically, we found that the TRIF adaptor protein is required for any synergistic enhancement of MyD88 (upstream) and interferon regulatory factor 5 (IRF5) (downstream)-dependent type I IFN and IL-12p70 cytokine response to MPLA-CpG-PLPs. Furthermore, we show that the kinetics and magnitude of downstream tumor necrosis factor (TNF) receptor-associated factor 6 (TRAF6) and IRF5 signaling events play an important role in driving the synergistic cytokine responses. These findings provide the fundamental mechanistic basis of the integrated response to TLR4-TLR9 dual engagement in BM-APCs and motivate kinetic signaling studies in the evaluation of innate immune cross-talk for various combinatorial adjuvant platforms.

\section{RESULTS \\ Density of CpG presentation, but not particle size, influences synergistic cytokine responses in BM-APCs treated with MPLA-CpG carrying PLPs}

To present MPLA and CpG adjuvants at the same time, we synthesized pathogen-like nanoparticles (NPs) and microparticles (MPs) (PLPs). The PLPs are poly(lactic-co-glycolic acid) (PLGA) particles with branched polyethylenimine (bPEI) conjugated to the particle surface. We have extensively published on the synthesis, characterization, and delivery of single and multiple adjuvants and antigens on these PLPs, both in vitro and in vivo $(19,31,33,34)$. For the current studies, MPLA adjuvant was first encapsulated into the particle during double-emulsion, solvent evaporation-based synthesis. The PLPs were then covalently modified with a monolayer of bPEI to impart a positive surface charge, which enables electrostatic loading of the negatively charged $\mathrm{CpG}$ adjuvants on the particle surface, thereby allowing dual loading of both MPLA and CpG. We synthesized two sizes of PLPs, MP sized and NP sized, with either single adjuvants ( $\mathrm{M}$ for MPLA and $\mathrm{C}$ for $\mathrm{CpG}$ ) or dual adjuvants (MPLA and $\mathrm{CpG}$ or MC) (Fig. 1A and tables S1 and S2). PLPs loaded with dual adjuvants at an MPLA-to-CpG target ratio of 1:10 induced a slightly higher synergistic immune response than particles with an MPLA-to-CpG target ratio of 1:1 (fig. S1, A and B). Therefore, throughout the rest of experiments, we used a 1:10 target ratio of MPLA to CpG. It should also be noted that the encapsulated MPLA dose was chosen to induce a minimal cytokine response so that we could study how baseline concurrent TLR4 signaling synergizes with the stronger TLR9 response. We used both liquid chromatographymass cytometry (LC-MS) and gas chromatography-mass spectrometry (GC-MS) methods to measure MPLA encapsulation efficiency in PLPs, which showed variable results between these two methods (table S3). In addition, as a surrogate method to verify MPLA encapsulation in PLPs, we encapsulated lipopolysaccharide (LPS)-fluorescein isothiocyanate (FITC) in PLPs and estimated the encapsulation efficiency by fluorometry, which showed different encapsulation levels from LC-MS or GC-MS (table S3). For CpG, however, we confirmed 100\% surface loading levels on the PLPs using nucleic acid quantification method.

Furthermore, the NPs were synthesized with two different adjuvant densities, i.e., mass of adjuvant per particle (high density, henceforth shown in figures and text as "Hi," and low density, shown in figures and text as "Lo") for both MPLA and CpG, while MPs were synthesized with low density for MPLA and high density of the $\mathrm{CpG}$ adjuvant. A high density of adjuvant was about sixfold higher than the low density of adjuvant. We did not have MPs with both high- and low-density combinations for MPLA and CpG (like for NPs) due to several technical reasons as explained in detail in table S1. Nevertheless, these PLP designs allowed us to compare both size effects at the same density and density effects at the same size. To reiterate, total target MPLA and/or CpG doses were always kept constant across all experimental and control groups in all studies.

We first studied how particle size and adjuvant density influenced the cytokine response in GM-CSF-differentiated mouse BM-APCs. Numerous previously published papers describe GMCSF-differentiated murine bone marrow cells as bone marrowderived dendritic cells (BMDCs); however, recent literature evidence suggests a heterogenous mixture of innate immune cells, including dendritic cells (DCs), macrophages, and monocytes present in the GM-CSF-differentiated bone marrow cells around 6 to 7 days of culture (35-37). Hence, we refer our GM-CSF-differentiated murine bone marrow cells as BM-APCs instead of BMDCs throughout this manuscript.

In BM-APCs, the IFN- $\beta$ response to MP (MP-MLo-CHi) and NP-Hi with both MPLA and CpG was synergistic when compared to the IFN- $\beta$ responses to single adjuvants. In contrast, there were minimal IFN- $\beta$ responses from NPs with low densities of MPLA and $\mathrm{CpG}$ adjuvants (Fig. 1B). The IL-12p70 responses to MP and NP-Hi with both MPLA and CpG mirrored those of the IFN- $\beta$ responses. A lower IL-12p70 response was observed after treatment with NPs with a low density of $\mathrm{CpG}$, and concurrent treatment with MPLA did not generate a synergistic response (Fig. 1C). We also measured IL-27, which is one of the members of IL-12 cytokine families. As with IL-12p70, IL-27 response was also synergistically enhanced in BM-APCs by MPLA-CpG-Dual PLPs (both MPs and NPs) with high CpG density (fig. S2). In addition, we measured the IL-6, TNF- $\alpha$, and IL-10 responses to PLPs with MPLA and CpG. IL-6 responses were primarily CpG driven in MPs, MPLA driven in NPs with low adjuvant density, and additive between MPLA and CpG in NPs with high adjuvant density (Fig. 1D). TNF- $\alpha$ secretion was not statistically different between the groups (Fig. 1E). However, for both IL-6 and TNF- $\alpha$, MPs showed higher responses compared with NPs (Fig. 1, D and E). As with IFN- $\beta$ and IL-12p70, the IL-10 response was synergistic for MPs and NPs with high densities of adjuvants but not synergistic for NPs with low densities of adjuvants (Fig. 1F). The effect of biophysical parameters of PLPs on IFN- $\beta$, IL-12p70, IL-27, IL-10, TNF- $\alpha$, and IL6 is summarized in table S4. 
A

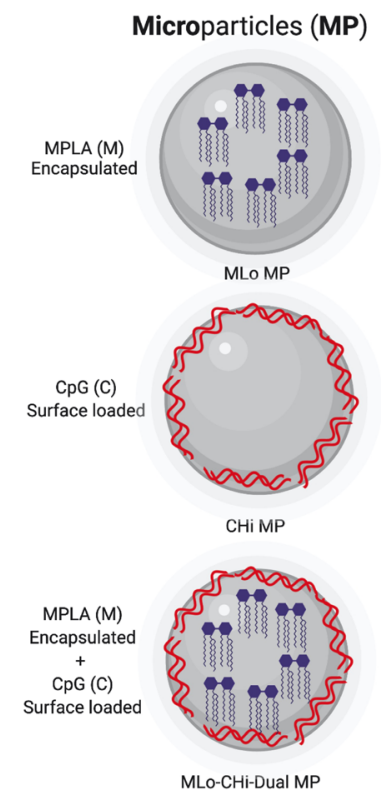

D

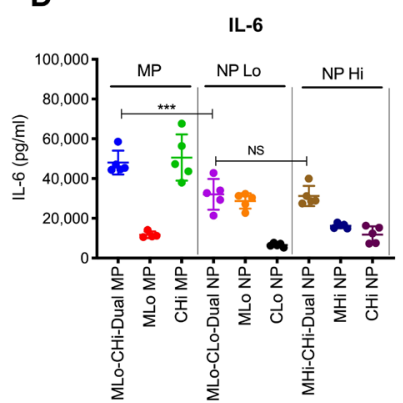

Nanoparticles (NP)

Low adjuvant density

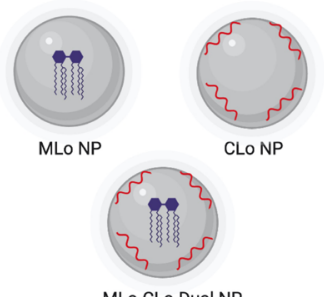

MLo-CLo-Dual NP

High adjuvant density

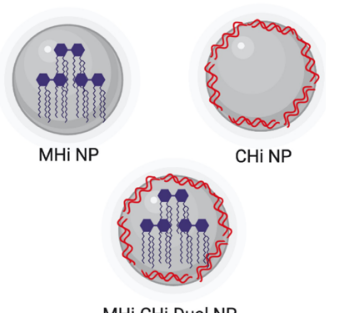

E

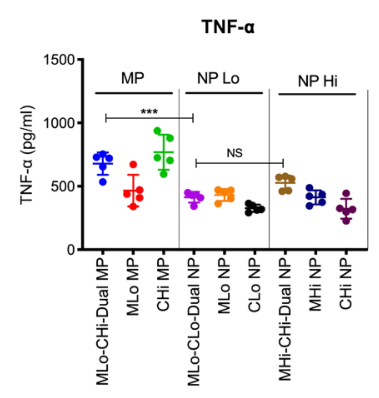

B

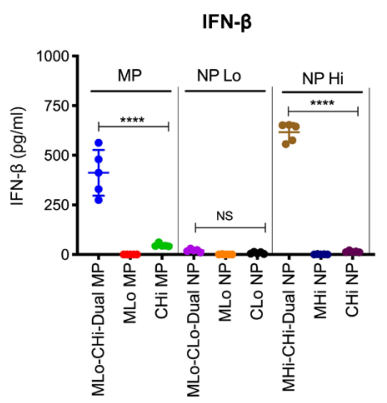

C

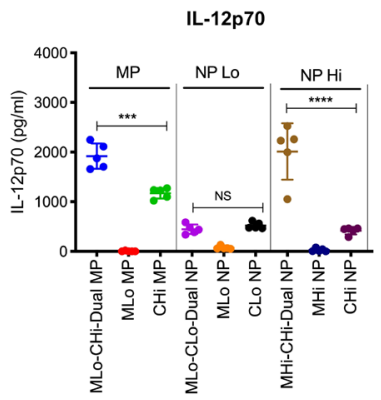

$\mathbf{F}$

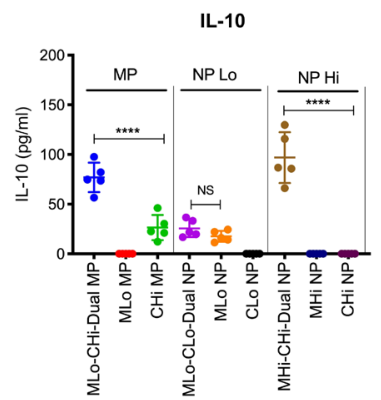

Fig. 1. Synergistic cytokine responses from BM-APCs induced by PLPs with MPLA and CpG depend on CpG adjuvant density. (A) Schematic of particle formulations for the co-delivery of MPLA and CpG. (B to F) Murine GM-CSF-differentiated murine BM-APCs $(300,000$ cells per well) were treated with formulations of varying size and CpG ligand density. IFN- $\beta$, IL-12p70, IL-6, TNF- $\alpha$, and IL-10 in cell supernatants 24 hours after treatment. Each data point represents an independently treated well $(n=5)$. Center lines designate the mean value, and error bars represent SD. ${ }^{* *} P<0.001$ and ${ }^{* * *} P<0.0001$; NS, not significant; one-way analysis of variance (ANOVA) with Tukey's multiple comparison test.

Next, we analyzed various immune cell subsets in GM-CSFderived BM-APCs and their intracellular cytokine levels following 6 hours of activation with PLPs using flow cytometry. DCs, macrophages, and monocytes were the major immune cell population in the GM-CSF-derived BM-APCs (figs. S3A, S4A, and S5A), and each of these immune cells showed differential levels of intracellular IL-12p70, TNF- $\alpha$, and IL- 6 cytokines upon activation with PLPs for 6 hours. For IL-12p70, DCs produced more cytokine than macrophages, and monocytes had the minimal level (fig. S3B). Furthermore, in both DCs and macrophages, MPLA-CpG dual and CpG single adjuvants (both for MPs and NPs) with CpG high density showed high IL-12p70 levels as compared to other groups (fig. S3B). For TNF- $\alpha$, macrophages are the major producers followed by DCs and monocytes (fig. S4B). Moreover, in macrophages, high-density $\mathrm{CpG}$ on dual and single MPs and NPs triggered more TNF- $\alpha$ response than any other particulate adjuvant groups (fig. S4B). For IL-6, both DCs and macrophages contributed at similar levels that were higher than the monocytes (fig. S5B). MPLA-CpG low-density dual NPs (MLo-CLo-Dual NP) contributed the highest IL-6 among all the adjuvant groups (fig. S5B).
Further, we evaluated MPLA-CpG-Dual PLP uptake by different immune cell subsets in GM-CSF-derived BM-APCs using a fluorophorelabeled CpG (IR700-CpG). All the major immune cell subsets in the BM-APCs, such as monocytes, macrophages, and DCs, showed uptake of both MPLA-CpG-Dual MPs and NPs (fig. S6). The overall trend of PLP uptake was similar across the cell types; however, MLo-CLo-Dual NP uptake showed higher levels [in terms of median fluorescent intensity (MFI) in macrophages and DCs as compared to the monocytes (fig. S6B)]. Further, MLo-CLo-Dual NP showed a higher level of uptake (in terms of MFI) than MLo-CHi-MP and MHi-CHi-NP across all the cell types (fig. S6C).

\section{The adaptor protein TRIF is required for the synergistic type IIFN and IL-12p70 response induced by dual MPLA-CpG PLPs in BM-APCs}

To identify why PLPs with MPLA and CpG induce synergistic IFN- $\beta$ and IL-12p70 responses, we systematically evaluated the signaling pathways driven by TLR4 and TLR9. Activation of TLR4 on the plasma membrane results in the recruitment of the adaptor protein 
MyD88. When MPLA-CpG-Dual PLPs are internalized, they can activate both TLR4 and TLR9 in the endosome. Endosomal TLR4 activation is known to recruit the adaptor protein TRIF, while endosomal TLR9 activation recruits the adaptor protein MyD88. Both TLR9 and TLR4 signaling, regardless of location, activate TRAF6, which is a central mediator for downstream signaling that ultimately triggers type I IFN and IL-12p70 (Fig. 2A).

Here, we first evaluated the effect of MyD88 and TRIF knockdown on the IFN- $\beta$ and IL-12p70 responses from BM-APCs induced by PLPs. With MyD88 ${ }^{-/-}$BM-APCs, we observed complete ablation of the IFN- $\beta$ and IL-12p70 response for MPLA-CpG-Dual MPs and NPs with high density for CpG, suggesting that MyD88 is the primary adaptor protein for the MPLA-CpG-Dual PLP adjuvant signaling (Fig. 2, B and C). In contrast, the synergistic increases in IFN- $\beta$ and IL- 12 p70 responses for MPLA-CpG-Dual MPs and NPs with high CpG density were lost in $\mathrm{TRIF}^{-/-}$BM-APCs, and the cytokine levels remained at the CpG-Hi single adjuvant level for MPs and NPs (Fig. 2, B and C). This indicates that TRIF adaptor protein is required only for the synergistic enhancement of IFN- $\beta$ and IL-12p70 responses by dual-loaded MPLA-CpG-PLPs. Furthermore, using BM-APCs from TLR $4{ }^{\text {mut }}$ mice, we also confirmed that synergistic IFN- $\beta$ and IL-12p70 production due to MPLA-CpGDual PLPs with high CpG density is dependent on TLR4 signaling from MPLA (fig. S7, A and B). However, with BM-APCs from TLR9 ${ }^{\text {mut }}$ mice, we observed synergistic enhancement of IFN- $\beta$ and IL-12p70 responses [at a lower level than wild-type (WT) BM-APCs] for Dual PLPs with high CpG density (fig. S7, A and B), which could be due to the lack of complete ablation of TLR9 gene in the TLR9 ${ }^{\text {mut }}$ mice. To demonstrate that $\mathrm{CpG}$ [oligodeoxynucleotide (ODN) 1826] signals via TLR9, we compared the effect of ODN 1826 negative control (ODN2138) and ODN 1826 on the synergistic IL-12p70 response for MPLA-CpG-Dual MPs. We observed the ablation of synergistic IL-12p70 level when negative control ODN 1826 was used instead of ODN 1826, which signals via TLR9 (fig. S7C).

A

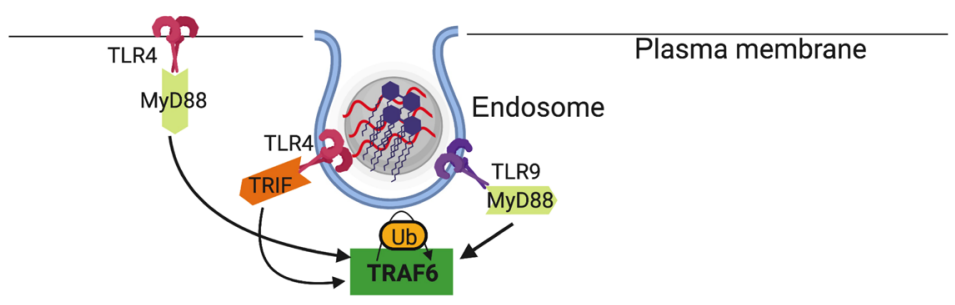

B

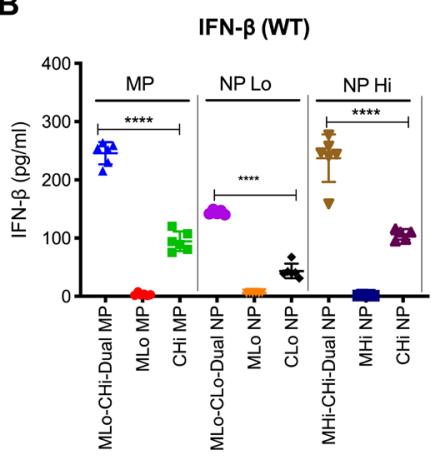

C

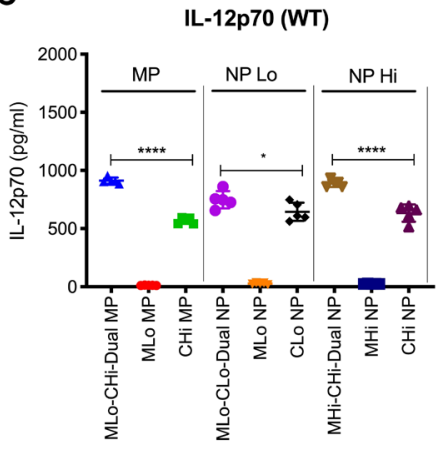

IFN- $\beta$ (MyD88 KO)

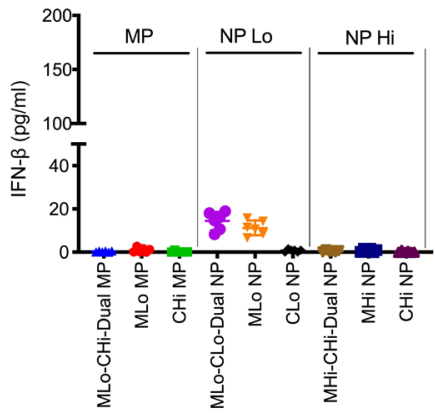

IL-12p70 (MyD88 KO)

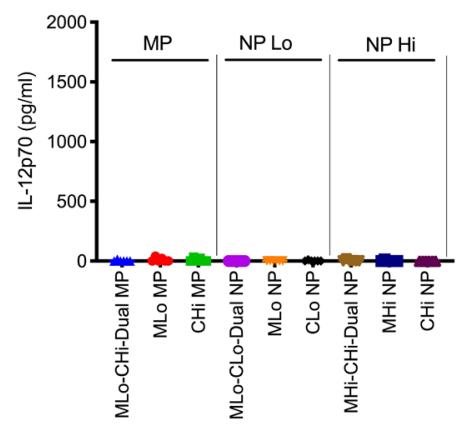

IFN- $\beta$ (TRIF KO)

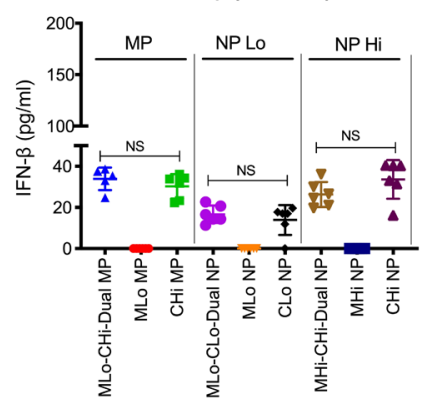

IL-12p70 (TRIF KO)

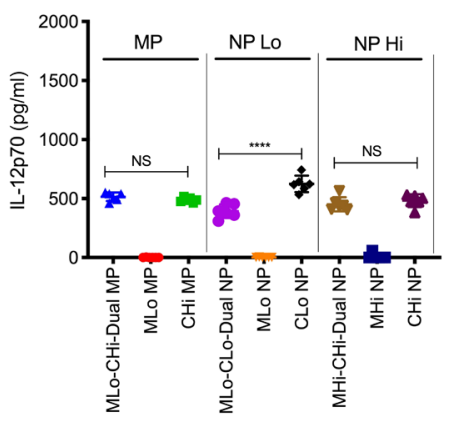

Fig. 2. Knockdown of the adaptor protein TRIF ablates synergy from PLPs with MPLA and CpG in BM-APCs. (A) Schematic showing early signaling through the adaptor proteins MyD88 and TRIF following activation of TLR4 and TLR9. (B) IFN- $\beta$ production from BMDCs derived from WT, MyD88 ${ }^{-/-}$, and TRIF ${ }^{-/-}$mice after PLP treatment. (C) IL-12p70 production from BM-APCs derived from WT, MyD88 ${ }^{-/-}$, and $\mathrm{TRIF}^{-/-}$mice after PLP treatment. Each data point represents an independently treated well ( $n=5$ to 6). Center lines designate the mean value, and error bars represent SD. ${ }^{*} P<0.05$ and ${ }^{* * *} P<0.0001$; NS, not significant; one-way ANOVA with Tukey's multiple comparison test. 


\section{IRF5, but not IRF 3 or IRF7, drives the innate immune response triggered by dual adjuvant-loaded MPLA-CpG PLPs in BM-APCs}

Activation of TLR4 and/or TLR9 induces TRAF6 ubiquitination, which proceeds to phosphorylate IRF3, IRF5, or IRF7 (Fig. 3A). To identify the most important IRF in downstream signaling, we measured IFN- $\beta$ and IL-12p70 in BM-APCs from IRF3, IRF5 [without DOCK2 gene mutation (38); fig. S8], and $\mathrm{IRF}^{-/-}$mice. The synergistic IFN- $\beta$ responses in IRF3 ${ }^{-1-}$ BM-APCs were higher than those in WT cells. In IRF5 ${ }^{-/-}$BM-APCs, IFN- $\beta$ responses were completely ablated regardless of PLP treatment. There were no differences in IFN- $\beta$ responses in IRF7 ${ }^{-/-}$BM-APCs when compared to WT (Fig. 3B). As with IFN- $\beta$, IL-12p70 responses in IRF3 ${ }^{-/-}$BM-APCs were increased when compared to WT. IL-12p70 responses were also ablated in IRF5 ${ }^{-1-}$ BM-APCs. In IRF7 ${ }^{-/-}$BM-APCs, IL-12p70 secretion was decreased when compared to WT (Fig. 3C). We further investigated whether IFN- $\beta$ increased IL-12p70 secretion by engaging IFN- $\alpha$ receptor (IFNAR) in an autocrine or paracrine way. In IFNAR ${ }^{--}$ BM-APCs, synergistic level of IL-12p70 decreased partially in MPLA-CpG-Dual MP and NP groups, suggesting that the autocrine or paracrine effect of IFN- $\beta$ has a partial effect on enhancement of IL-12p70 production via IFNAR. Notably, we observed significantly higher IFN- $\beta$ levels in IFNAR ${ }^{-/}$BM-APCs compared to WT BM-APCs, likely as a result of accumulation of unused IFN- $\beta$ in the culture medium in BM-APCs from IFNAR ${ }^{-/-}$mice (fig. S9, A and B).

\section{Sustained and elevated IRF5 phosphorylation and TRAF6 levels are responsible for synergistic innate immune responses by MPLA-CpG-Dual PLPs in BM-APCs}

TRAF6 ubiquitination activates a sequence of kinases, which ultimately leads to IRF5 phosphorylation. This event enables activated IRF5 to translocate from the cytoplasm into the nucleus, where it can initiate transcription of mRNA encoding for IFN- $\beta$ and proinflammatory cytokine IL-12p70 (Fig. 4A). Given that we found that IRF5 is primarily responsible for the dual adjuvant signaling, we further investigated what aspect of IRF signaling, amplitude or kinetics or both, is responsible for the synergistic cytokine production. To evaluate this, we measured total IRF5 and phosphorylated IRF5 protein levels over time. After MPLA-MP (single adjuvant) treatment, phosphorylated IRF5 levels were highest after $30 \mathrm{~min}$ to 1 hour. No phosphorylated IRF5 was detected after CpG-MP treatment. Phosphorylated IRF5 levels were high between $30 \mathrm{~min}$ and 4 hours after MPLA-CpG-Dual MP treatment (Fig. 4B), indicating prolonged signaling. In agreement with phosphorylated IRF5 levels, the rate of IRF5 translocation to the nucleus peaked 4 hours after treatment (Fig. 4C). The ratio of nuclear to cytoplasmic IRF5 in BM-APCs treated with MPLA-CpG-Dual MP is approximately five times higher than the ratio in BM-APCs treated with CpG-MP (Fig. 4C). A second surge of IRF5 translocation from the cytoplasm to the nucleus occurs at 24 hours. We also assessed the kinetics of IFN- $\beta$ and IL-12p70 production by BM-APCs of PLP adjuvants and observed synergistic increase in the cytokine production for MPLACpG-Dual MP, which peaked at around 6 hours and sustained over 24 hours after stimulation with MPLA-CpG-Dual MPs (Fig. 4D).

Next, we measured the expression kinetics of TRAF6, which is the master regulator that drives IRF5, NF- $\mathrm{\kappa B}$, and AP-1 (activating protein 1) signaling and ultimately leads to production of type I IFN and proinflammatory cytokines, including IL-12p70. We hypothesized that sustained and elevated TRAF6 signaling upstream to
IRF5 could lead to synergistic IFN- $\beta$ and IL-12p70 responses in BM-APCs activated by MPLA-CpG-Dual MP (Fig. 4E). In BM-APCs treated with MPLA-MP, total TRAF6 expression peaked at $30 \mathrm{~min}$ after treatment and disappeared after 4 hours. For BM-APCs treated with CpG-MP, total TRAF6 expression peaked at 4 hours and declined by 6 hours. For BM-APC-treated MPLA-CpG-Dual MP, sustained and elevated TRAF6 expression was observed over 24 hours (Fig. 4E), which indicates that kinetics of TRAF6 signaling is correlated with cytokine synergy.

Overall, as shown in Fig. 5, we identified the key signaling mediators that play critical roles in driving synergistic IFN- $\beta$ and IL-12p70 cytokine responses induced by co-presentation of the TLR4 adjuvant MPLA and the TLR9 adjuvant CpG on synthetic PLPs. MPLACpG-Dual PLPs activate both TLR4 and TLR9 in the endosome that subsequently engage TRIF (primarily from MPLA) and MyD88 (primarily from $\mathrm{CpG}$ ) adaptor and lead to sustained and elevated TRAF6 expression and IRF5 phosphorylation and nuclear translocation. Last, sustained and elevated level of IRF5 causes synergistic production of IFN- $\beta$ and IL- $12 p 70$. Secreted IFN- $\beta$ binds to IFNAR and further boosts IL-12p70 production by autocrine/paracrine signaling.

In addition, we assessed IFN- $\beta$ and IL-12p70 responses of our PLPs in fms-like tyrosine kinase 3 (FLT3)-derived BM-APCs from WT and IRF5 knockout (KO) mice. In WT FLT3-BM-APCs (fig. S10A), we did not observe any synergistic IFN $-\beta$ responses for any of the MPLA-CpG-Dual PLP groups, and the IFN- $\beta$ responses were majorly driven by $\mathrm{CpG}$ when specifically delivered on NPs, rather than on MPs. MPLA-CpG dual adjuvant NP groups had lower response than CpG single adjuvant NP groups. Similarly, we did not observe any synergistic response for IL-12p70 for any of the dual adjuvant groups (fig. S10B). For both IFN- $\beta$ and IL-12p70, NPs with high CpG density showed higher responses than NPs with low CpG density or MPs with high CpG density groups. Furthermore, IRF5 KO BM-APCs mirrored WT BM-APCs with similar levels of IFN- $\beta$ and IL-12p70 across PLPs (fig. S10, C and D). Our flow cytometry analysis of immune cell subsets in BM-APCs suggests that FLT3-derived BM-APCs are composed of plasmacytoid DCs, conventional DCs (cDC1 and $\mathrm{cDC} 2$ ), and monocytes (fig. S10E), whereas GM-CSF-derived BM-APCs contain majorly cDCs, macrophages, and monocytes (figs. S3A, S4A, and S5A).

\section{DISCUSSION}

We investigated the effect of biophysical properties such as size and adjuvant density on the efficiency of synthetic particulate carriers (PLPs) to induce synergistic innate immune responses through concurrent activation of TLR4 and TLR9 in GM-CSF-derived BM-APCs. In addition, we investigated how specific intracellular signaling pathways drive synergistic innate immune responses through TLR4 and TLR9 cross-talk. This synergistic phenomenon has been identified in multiple reports by our group and others $(7,21,31,39)$. Others have reported that in neutrophils, MPLA and CpG dual delivery may trigger TLR9 translocation from the cytosol to the endosome by the initial TLR4 activation (32). Despite evidence of such synergy, the underlying signaling mechanism and the specific signaling molecules involved in driving MPLA-CpG (TLR4/TLR9) dual adjuvant synergy in APCs have not yet been fully elucidated. Here, we evaluated each of the intermediate steps in the TLR4 and TLR9 signaling pathways in GM-CSF-derived BM-APCs to identify whether and how they contribute to synergistic type I IFN and IL-12 responses. 
A

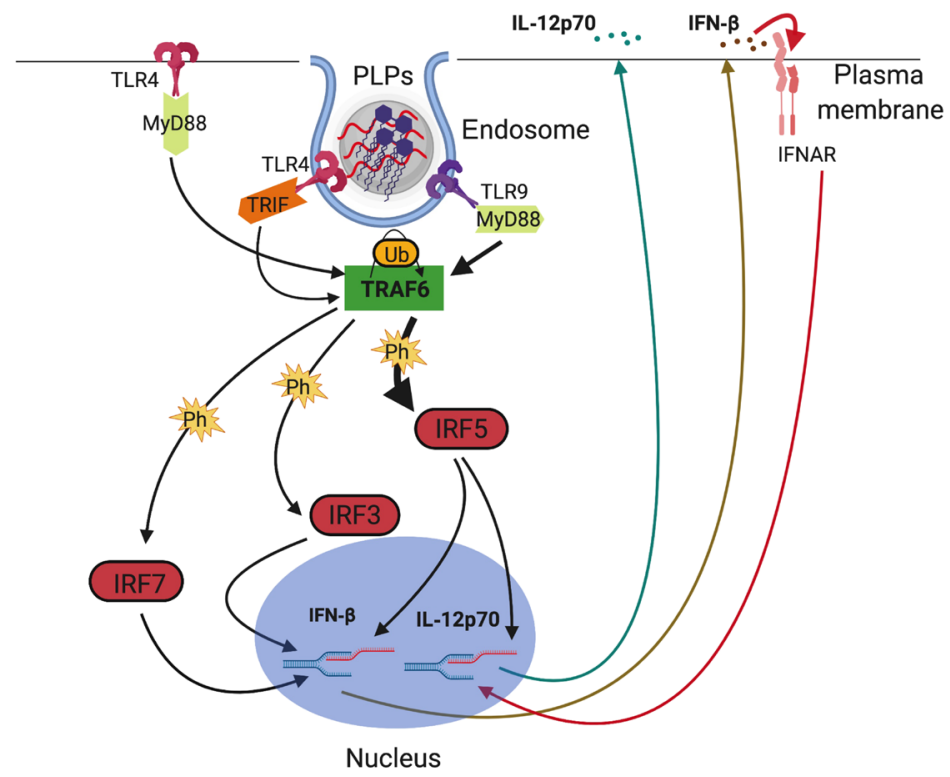

B
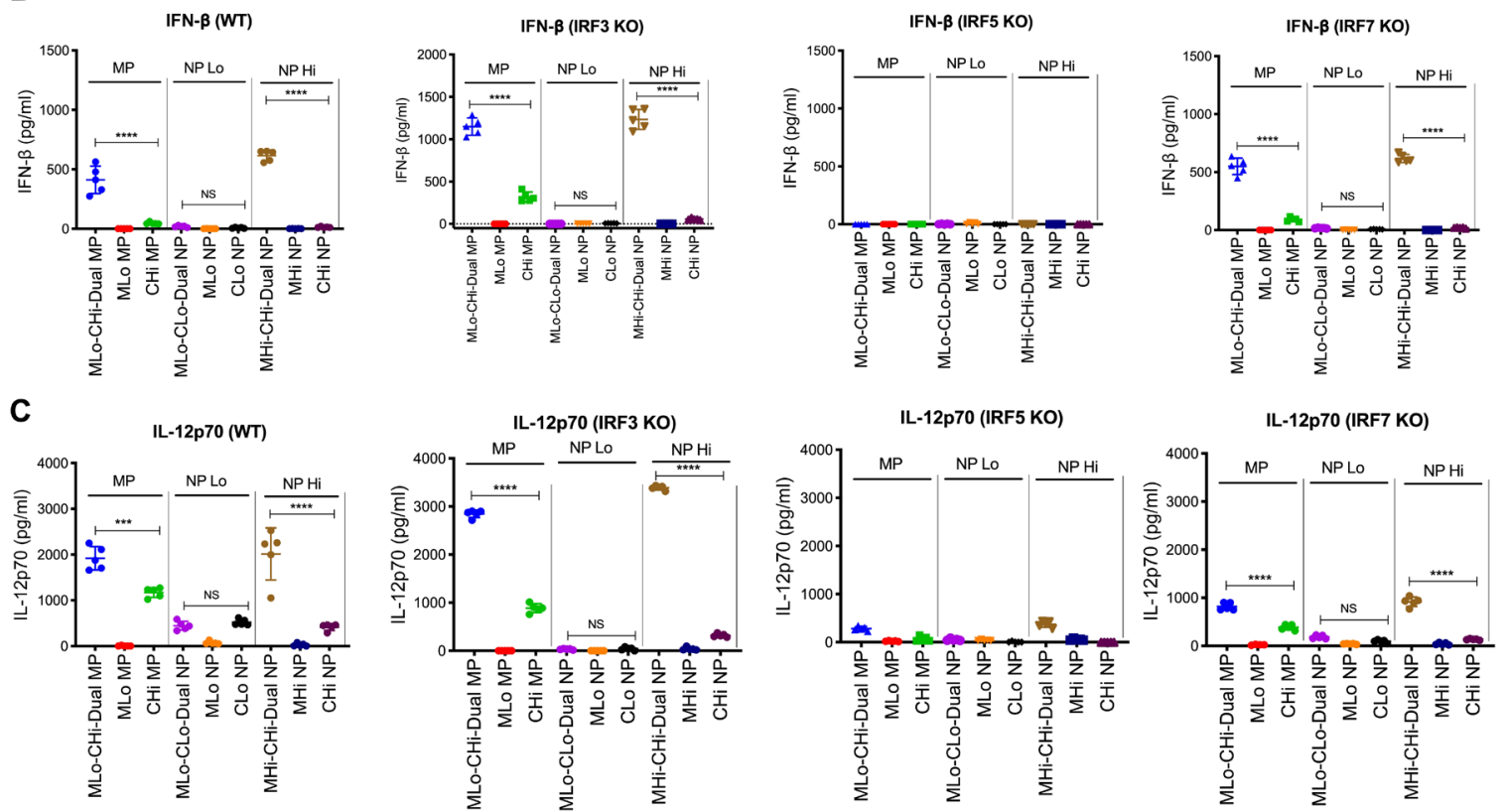

Fig. 3. IRF5, not IRF3 or IRF7, drives innate immune response triggered by MPLA and CpG in BM-APCs. (A) Diagram of TLR4 and TLR9 downstream signaling. (B) IFN- $\beta$ production from BM-APCs derived from WT, IRF $3^{-/-}$, IRF5 $5^{-/-}$, and IRF7 ${ }^{-1-}$ mice. (C) IL-12p70 production from BM-APCs derived from WT, IRF ${ }^{-1-}$, IRF5 ${ }^{-/-}$, and IRF7 $^{-1-}$ mice. We used the same WT BM-APCs in Fig. 1 for WT IL-12p70, IFN- $\beta$, TNF- $\alpha$, IL-6, and IL-10 responses. Each data point represents an independently treated well ( $n=5$ ). Center lines designate the mean value, and error bars represent SD. ${ }^{* *} P<0.001$ and ${ }^{* * * *} P<0.0001$; NS, not significant; one-way ANOVA with Tukey's multiple comparison test.

We have previously shown that increasing surface density of CpG (as a single adjuvant) on PLPs increased IL-12p70 production from BM-APCs (33). Here, we found that the adjuvant density effect also applies to IFN- $\beta$ and IL-12p70 responses from dual TLR4/TLR9 engagement. NPs with a high MPLA and CpG density triggered synergistic immune responses in BM-APCs, while NPs with low MPLA and CpG density triggered a low IL-12p70 response and no detectable IFN- $\beta$ response in BM-APCs. The NPs with high MPLA and CpG density, however, triggered equivalent IFN- $\beta$ and IL-12p70 responses to MPs with high CpG but low MPLA density.
The equivalent responses to MPs and NPs with matched $\mathrm{CpG}$ density indicate that the property of $\mathrm{CpG}$ density plays a dominant role in driving IFN- $\beta$ and IL-12p70 responses. Notably, MPLACpG-Dual PLPs also showed CpG density-dependent IL-27 response similar to IL-12p70, suggesting activation and secretion of multiple cytokines from IL-12 cytokine families. IL-27 is an important cytokine that has important roles in vaccine-induced $\mathrm{T}$ cell response, in terms of magnitude and memory response (40). We also saw strong synergy for IL-10 production, similar to IL-12 and IFN- $\beta$, and although MPs outperformed NPs for CpG production 
A

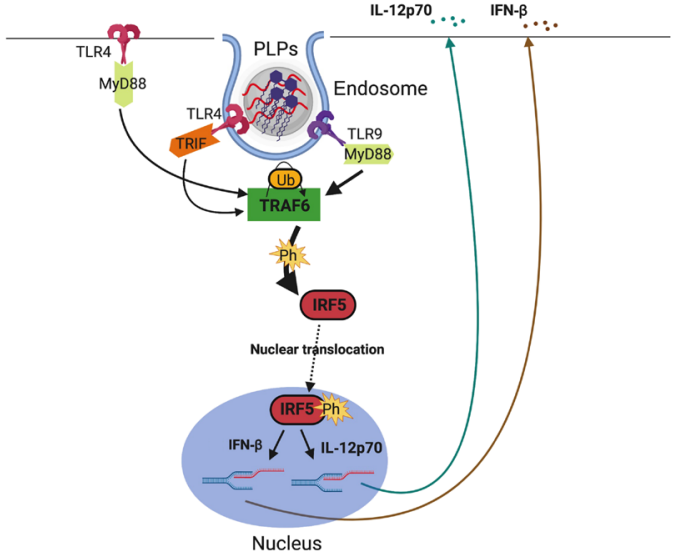

B
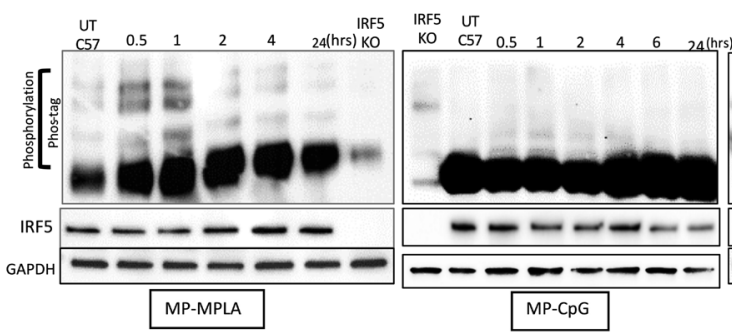

IRF5 UT

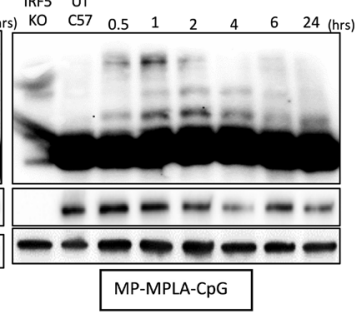

C
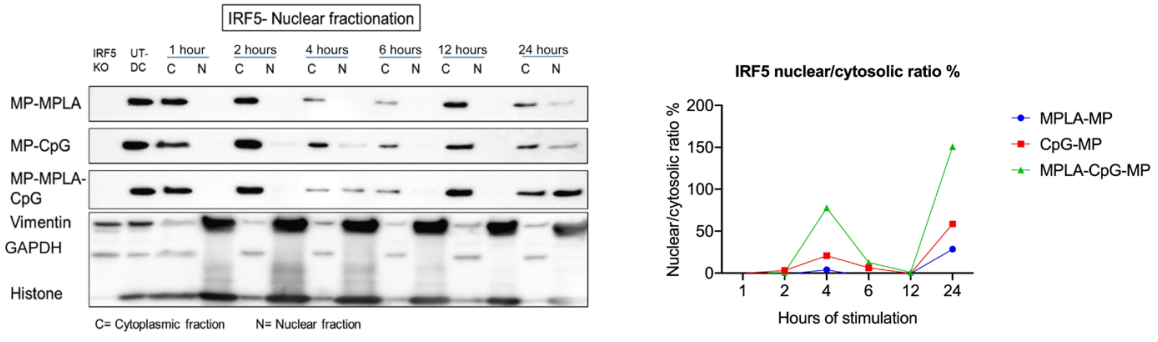

D
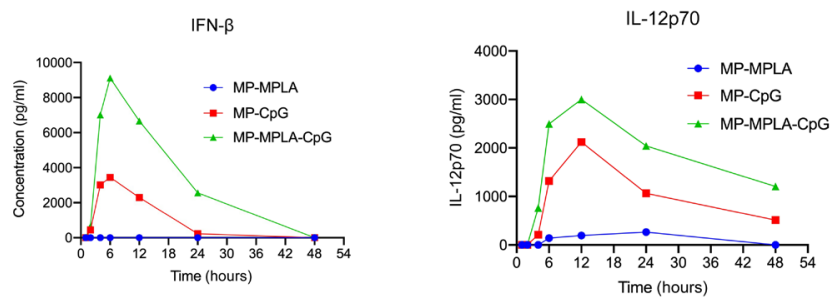

E
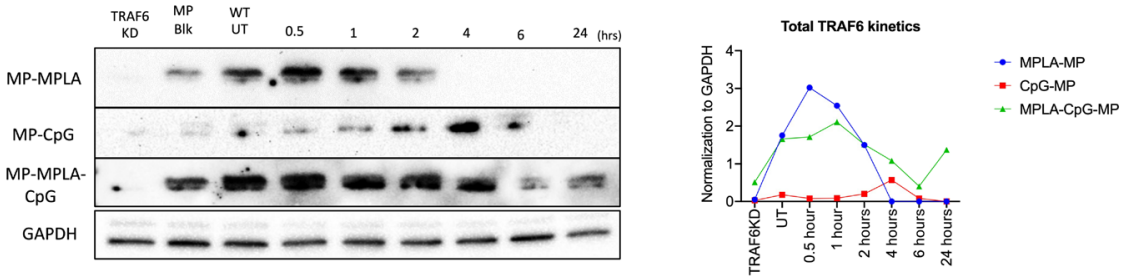

Fig. 4. Sustained IRF5 phosphorylation, higher IRF5 nuclear translocation, and elevated TRAF6 are responsible for synergistic IFN and IL-12p70 responses in BM-APCs. (A) Schematic showing TRAF6 and IRF5 signaling following TLR4 and TLR9 activation. (B) Levels of phosphorylated (Phos-tag) and total IRF5 [SDS-polyacrylamide gel electrophoresis (PAGE)] after 0.5, 1, 2, 4, 6, and 24 hours of BM-APCs treated with MPs with MPLA and/or CpG. GAPDH (glyceraldehyde-3-phosphate dehydrogenase) was used as a loading control, and treated IRF5 ${ }^{-/-}$BM-APCs were used as negative controls. (C) Total IRF5 levels in the nuclear and cytoplasmic fractions at $1,2,4,6,12$, and 24 hours of BM-APC treated with MPs with MPLA and/or CpG. Ratio of nuclear-to-cytoplasmic IRF5 after 1,2, 4, 6, 12, and 24 hours of treatment, where a higher ratio indicates a higher rate of nuclear translocation. Ratios were performed by Bio-Rad Image Lab software. (D) Kinetics of IFN- $\beta$ and IL-12p70 production by BM-APCs treated with PLPs. BM-APCs from this experiment were used for nuclear fractionation studies, and the results are shown in (C). (E) Levels of total TRAF6 (SDS-PAGE) after 0.5, 1, 2, 4,6 , and 24 hours of BM-APCs treated with MPs with MPLA and/or CpG. GAPDH was used as a loading control, and TRAF6 knocked down in BM-APCs was used as a negative control. On the right is the graphical representation of the GAPDH normalization of each blot. This was done with Bio-Rad Image Lab software. 


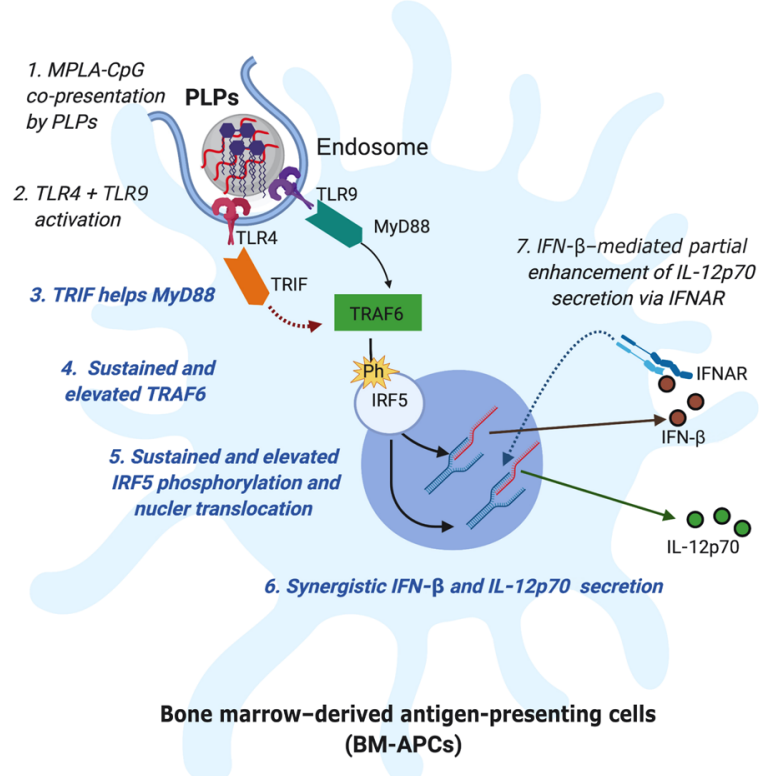

Fig. 5. Schematic showing the mechanism of synergistic IFN- $\beta$ and IL-12p70 cytokine response because of MPLA and CpG co-presentation on PLPs in BM-APCs.

even when densities were matched, the synergistic response was driven by adjuvant density rather than particle size. For IL- 6 and TNF- $\alpha$ responses, we saw that particle size had a stronger effect than adjuvant density. There was no synergy in dual delivery, and for both cytokines, MPs were much more effective for CpG single adjuvant delivery than NPs, regardless of CpG density. This difference was also similar for dual adjuvant delivery. Furthermore, MPLA alone induced significant IL- 6 and TNF expression, unlike what we saw for IFN- $\beta$ and IL-12; and for lower density of MPLA delivery as a single adjuvant, NPs outperformed MPs in IL-6 production. Together, these results further underscore the critical role of the biophysical properties of adjuvant presentation to APCs. Specifically, particle size and adjuvant density provide an additional way to fine-tune innate immunity induced by various TLR ligands, either as a mono-adjuvant or as combination adjuvants.

Notably, our flow analyses suggest that GM-CSF-derived BMAPCs contain a heterogenous mixture of DCs, macrophages, and monocytes, which upon activation (for 6 hours with brefeldin A for final 4 hours with PLPs) showed differential levels of intracellular cytokines, indicating that these cell types may contribute at different levels to the overall 24-hour secreted cytokine levels for our PLPs. However, it is important to note that the blocking of cytokine secretion by brefeldin A (required for intracellular staining) ablates autocrine and paracrine signaling of secreted cytokines (41), and thus, these results may not be directly comparable to the secreted 24-hour cytokine profiles of PLPs. In addition, the kinetics of secretion following particulate adjuvant treatment could be different for various cytokines, which limits our ability to directly compare our 6-hour intracellular cytokine results with 24-hour cumulative secreted cytokine results. Further, even if the PLP uptake was similar across DCs, macrophages, and monocytes, these immune cells triggered differential cytokine responses when activated by PLPs, indicating the diverse roles of these immune cells in their response to the specific adjuvant combination and PLPs.
For mechanistic understanding of the large synergy seen in type I IFN and IL-12p70 responses to dual presentation of MPLA and $\mathrm{CpG}$, we first evaluated whether the adaptor proteins that are commonly known to play major roles in TLR4 and TLR9 individual signaling are playing a critical role in developing the synergistic immune response in BM-APCs. TLR4 is present in both cell surface membranes and in the endosomal membranes. On the cell surface, TLR4 activation by LPS or MPLA leads to the recruitment of the adaptor protein MyD88 and its associated myddosome proteins (42). In the endosome, TLR4 activation results in the recruitment of the TRIF adaptor protein, which signals through receptor-interacting serine/ threonine-protein kinase 1 (RIPK1) to activate TRAF6. Both MyD88 and TRIF are critical to maximize TLR4-mediated DC maturation (43). TLR4-MyD88 interactions on the plasma membrane result in early innate immune activation, while TLR4-TRIF interactions in the endosome result in delayed innate immune activation (44). In this study, we observed that MyD88 is the primary adaptor protein for TLR9-driven signaling by CpG, whereas TRIF and TLR4 are both required for IFN- $\beta$ and IL-12p70 synergistic responses to MPLA-CpG dual engagement. This indicates that PLPs, which are rapidly ingested by APCs, may activate endosomal TLR signaling, and the dual presence of TLR4 and TLR9 in the endosomes drives synergistic behavior. It should also be noted that cell surface TLR4 signaling is expected to be low at early time points because MPLA is encapsulated inside the particles, while acidic environments (as in endo/lysosomes) can accelerate PLGA degradation (45) and release MPLA more rapidly. In TLR9 ${ }^{\text {mut }}$ BM-APCs, we observed synergistic responses from MPLA and CpG treatment that were lower than observed from WT BM-APCs. The TLR9 ${ }^{\text {M7NBttr/MmJax }}$ (TLR9 $^{\text {mut }}$ ) strain has a chemically induced single point mutation that prevents TNF- $\alpha$ production after CpG stimulation in macrophages (46). Given that TLR9 is mutated and not completely knocked down in this strain, it is likely that high doses of $\mathrm{CpG}$ can still trigger low amounts of TLR activation.

We showed that IRFs downstream play a role in MPLA- and CpG-mediated signaling. Knockdown of IRF3 in BM-APCs amplified IFN- $\beta$ and IL- 12 p70 responses to MPLA and CpG. IRF3 is known to inhibit the binding of IRF5 to IL-12 promoters, so IRF3 knockdown may potentiate the magnitude of IRF5 signaling, causing higher IFN- $\beta$ and IL- 12 p70 response in IRF ${ }^{-/-}$BM-APCs (47). IRF7 knockdown did not affect the magnitude of the IFN- $\beta$ response but approximately halved the magnitude of the IL-12p70 response. In the IRF7 ${ }^{-1-}$ BM-APCs, synergistic responses were still observed for both IFN- $\beta$ and IL-12p70. Last, and most importantly, IFN- $\beta$ and IL-12p70 responses to both individual and combination adjuvants were ablated in IRF5 ${ }^{-/-}$BM-APCs. IRF5 knockdown has been shown to down-regulate $\mathrm{CpG}$-mediated proinflammatory cytokine production in hematopoietic cells (48). We also observed that knockdown of IFNAR results in an increase in levels of secreted IFN- $\beta$ and IL-12p70. IFNAR typically internalizes free type I IFNs to perpetuate downstream signaling, so the absence of IFNAR would result in accumulation of extracellular IFN- $\beta$ in the closed in vitro system where our experiments were conducted.

An in-depth analysis of IRF5 signaling revealed that synergistic responses to TLR4 and TLR9 activation are associated with prolonged signaling kinetics. After treatment with PLPs, we evaluated IRF5 phosphorylation, an essential event for nuclear translocation and subsequent transcription for type I IFNs and proinflammatory cytokines (e.g., IL-12p70). MPLA-MP up-regulated levels of phosphorylated 
IRF5 in the window of 30 min to 1 hour after treatment of BM-APCs. Phosphorylation of IRF5 was delayed in response to MP-CpG; heightened levels of phosphorylated IRF5 were not observed until 24 hours after treatment. Notably, MPs with both MPLA and CpG up-regulated levels of phosphorylated IRF5 from $30 \mathrm{~min}$ to 4 hours. This indicates that adjuvants with different signaling latency can be combined to form a system that drives a longer-lasting immune response. We found that prolonged IRF5 signaling was associated with a higher rate of IRF5 nuclear translocation. Analysis of fractionated lysates showed that nuclear IRF5 levels were significantly higher after treatment with MPLA and CpG than with either adjuvant alone. The time at which peak translocation occurs, 4 hours, is in agreement with studies evaluating NOD2 (nucleotide-binding oligomerization domain-containing protein 2) stimulation (49). Together, these data suggest that both prolonged phospho-IRF5 expression and increased amount of nuclear translocation are likely causes for the cytokine synergy observed.

Further, we studied the signaling kinetics of TRAF6 as it bridges TLR4 and TLR9 activation upstream with IRF5 signaling downstream. We found that TRAF6 followed similar patterns (kinetics and magnitude of signaling) as IRF5 signaling for MPs with MPLA, CpG, and dual adjuvants. MPLA-stimulated BM-APCs up-regulated at $30 \mathrm{~min}$ to 1 hour, while treatment with $\mathrm{CpG}$ peaked at 4 hours. Dual MPLA and CpG lead to a prolonged expression from $30 \mathrm{~min}$ to 24 hours, as seen in protein levels via Western blot. Overall, as with IRF5 signaling, elevated and sustained TRAF6 signaling from dual MPLA and CPG adjuvants aided in the synergistic cytokine response.

Previously, using a combination of soluble TLR adjuvants, such as LPS and CpG or poly I:C (polyinosinic:polycytidylic acid) and $\mathrm{CpG}$, Ouyang et al. (50) reported a synergistic increase in IL-12p40 cytokine in mouse peritoneal macrophages via MyD88-TRIF-IRF5 pathways. Our results show that in mouse BM-APCs, which is a mixture of BMDCs, macrophages, and monocytes, a similar signaling axis is active and provides synergistic enhancement of both type I IFN- $\beta$ and IL-12p70 when MPLA and CpG are co-presented using synthetic polymer-based PLPs. In this study, we identified the critical role of signaling kinetics and adjuvant density as key mediators of the synergy. These results can have major implications in vaccine design given the relevance of DCs, monocytes, and macrophages in eliciting vaccine responses and the potential of controlling synergistic responses by manipulating the biophysical properties of the adjuvant presentation. Notably, when FLT3-derived BM-APCs were activated with our PLPs, we did not observe any synergistic IFN- $\beta$ and IL-12p70 responses and involvement of IRF5 pathway for these cytokine as we observed with the GM-CSF-derived BM-APCs. FLT3 and GM-CSF-derived APCs are composed of different types of immune cell subsets, which have differential cytokine responses across various PLPs and may use IRF5 signaling pathway differently. Further in-depth investigation is needed to characterize differential cytokine responses and their specific pathways for these immune cell subsets when activated by PLPs, which is out of the scope of our current studies.

Investigating innate immune responses for pathogen-like copresentation of multiple PAMPs is an essential step in elucidating key components that modulate the host immune response against pathogens and allows us to develop better vaccines. Here, we explored the effect of biophysical properties (adjuvant density and size) of MPLA-CpG-Dual PLPs on synergistic cytokine responses in $\mathrm{BM}$-APCs in our study. Our results indicate that the density of $\mathrm{CpG}$ on PLPs is the main driver governing the synergistic IFN- $\beta$ and
IL-12 response seen, while PLP size also plays an important role in the production of other cytokines. Furthermore, our examination of TLR4 and TLR9 signaling pathways individually and in combination via adjuvant co-loaded PLPs yielded critical insights on how key intermediary proteins drive synergistic cytokine responses. Through gene $\mathrm{KO}$ studies, and protein and cytokine analyses down the signaling cascade, we found that the adaptor protein TRIF is required for any synergy, and downstream sustained and elevated TRAF6 signaling and IRF5 phosphorylation are the key drivers for the magnitude of the synergistic cytokine response for MPLA and CpG co-presentation on PLPs. Overall, we have identified the principal signaling components (TRIF-TRAF6-IRF5) that drive synergistic cytokine response for PLP-presented MPLA and CpG adjuvant combination in BM-APCs.

\section{MATERIALS AND METHODS \\ Study design}

In this study, we co-presented two clinically relevant TLR-based adjuvants, MPLA (TLR4 agonist) and CpG (TLR9 agonist), on our PLP- to GM-CSF-differentiated (7-day culture) BM-APCs to understand the effect of biophysical properties of PLPs on the synergistic innate immune response of MPLA-CpG dual adjuvants. Further, our overarching goal was to identify key molecular mediators and mechanistically understand their roles for the synergistic innate immune response of MPLA-CpG-Dual PLPs in BM-APCs. We synthesized polymeric MPs and NPs and co-loaded with MPLA (encapsulation) and CpG (surface loading) at different densities (high and low) and treated BM-APCs with the same MPLA-to-CpG dose ratio (1:10) across various PLP formulations. Using BM-APCs from WT mice, we examined the effect of biophysical properties on PLPs with MPLA and/or CpG on multiple proinflammatory cytokine responses, including IL-12p70 and IFN- $\beta$. Further, we used BM-APCs from different TLR4 and TLR9 signaling pathwayspecific gene knockout mice to identify key molecular mediators of synergistic IL-12p70 and IFN- $\beta$ response induced by MPLA-CpGDual PLPs. Last, we studied the signaling kinetics in WT BM-APCs using Western blot techniques to understand the molecular mechanism for the synergistic innate immune response for MPLA-CpG co-presentation on PLPs.

\section{Animals}

All animal experiments were conducted in accordance to approved IACUC (Institutional Animal Care and Use Committee) protocols by the Georgia Institute of Technology and Emory University. Female, 8- to 12-week-old C57/Bl6 mice (The Jackson Laboratory, Bar Harbor, ME) were used for all WT studies. IRF3 ${ }^{-1-}$, IRF5 ${ }^{-1-}$, $\mathrm{IRF}^{-/-}, \mathrm{MyD}^{-/-} 8^{-\mathrm{TRIF}^{-/-} \text {, and IFNAR }}{ }^{-/-}$mice were bred and housed at Emory University. TLR $4^{\text {lps-del//thJ }}$ and TLR9 ${ }^{\mathrm{M} 7 \mathrm{Btr} / \mathrm{Mm} / \mathrm{Mx}}$ mice (henceforth referred to as TLR $4^{\text {mut }}$ and TLR9 ${ }^{\text {mut }}$ mice; see Supplementary Methods for detailed description for these mice) were also purchased from The Jackson Laboratory and subsequently housed at Emory University.

\section{Mouse BM-APC culture}

Bone marrow was harvested from the tibias and fibulas of C57/Bl6 mice ( 6 to 10 weeks; The Jackson Laboratory, Bar Harbor, ME). The bone marrow cells were processed through a $40-\mu \mathrm{m}$ cell strainer, treated with RBC (red blood cell) lysis buffer, and seeded in petri 
dishes at a concentration of $1,000,000$ cells $/ \mathrm{ml}$ (total 20 million cells per petri dish). BM-APCs were differentiated from bone marrowderived cells through culture in Gibco RPMI 1640 medium (Thermo Fisher Scientific, Waltham, MA) with 10\% characterized fetal bovine serum (FBS; HyClone, Logan, UT), 1\% penicillin-streptomycin, $2 \mathrm{mM}$ glutamine, $1 \times \beta$-mercaptoethanol, $1 \mathrm{mM}$ pyruvate, and mouse recombinant GM-CSF (20 ng/ml; PeproTech, Rocky Hill, NJ). Ten milliliters of medium (50\% of initial volume in petri dish) was replaced with fresh medium supplemented with GM-CSF (40 ng/ml) on days 2 and 4. On day 6, $10 \mathrm{ml}$ of GM-CSF-supplemented (at $60 \mathrm{ng} / \mathrm{ml}$ ) medium was added without replacing any medium. On day 7, BM-APCs were harvested and replated in fresh culture medium supplemented with GM-CSF at $20 \mathrm{ng} / \mathrm{ml}$ for further experiment using PLP adjuvants. For FLT3-derived BM-APCs, bone marrow cells were cultured with human FLT3 ligand (PeproTech, Rocky Hill, NJ) at $200 \mathrm{ng} / \mathrm{ml}$ for 9 days without any change of medium. The cells were plated at 2 million cells per $\mathrm{ml}$ of medium and $5 \mathrm{ml}$ of medium per well in a six-well plate. At day 9, loosely adherent cells were harvested with vigorous pipetting and plated in fresh medium for further experiments.

\section{PLP synthesis and characterization}

TLR9 adjuvant ODN 1826 was purchased from InvivoGen (San Diego, CA). MPLA (Salmonella minnesota R595, catalog no. 699200P, and MPLA-PHAD, synthetic, catalog no. 699800) was purchased from Avanti Polar Lipids (Alabaster, AL). PLGA MPs and NPs were synthesized using a double-emulsion method, as reported previously by us $(33,51)$. Resomer RG $502 \mathrm{H}$ Poly(D-lactide-co-glycolide) (SigmaAldrich, St. Louis, MO) was dissolved in dichloromethane, and water was added and homogenized to form a primary emulsion. For MPLA formulations, MPLA was added to the dichloromethane before emulsification. MPs were formed through homogenization at $10,000 \mathrm{rpm}$ for $2 \mathrm{~min}$. The secondary emulsion was formed by adding the primary emulsion to $1 \%$ polyvinyl alcohol ( 87 to $89 \%$ hydrolyzed; Sigma-Aldrich) solution and by homogenizing at 10,000 rpm for $2 \mathrm{~min}$. NPs were formed through sonication at $65 \%$ power for $2 \mathrm{~min}$. The secondary emulsion was formed by adding the primary emulsion to a solution of $5 \%$ polyvinyl alcohol ( 87 to $89 \%$ hydrolyzed; Sigma-Aldrich) and by sonicating at $65 \%$ power for $5 \mathrm{~min}$. Dichloromethane was removed through rotary evaporation for 3 hours. PLGA MPs were pelleted by centrifugation for $20 \mathrm{~min}$ at $3000 \mathrm{~g}$ and washed with deionized (DI) water two times. PLGA NPs were pelleted by ultracentrifugation at $22,000 \mathrm{~g}$ for $20 \mathrm{~min}$ and washed with DI water two times. Both MPs and NPs were surface-modified with bPEI $\left(M_{\mathrm{W}}=70,000\right.$; Polysciences, Warrington, PA) through reaction via EDC [1-ethyl-3-(3-dimethylaminopropyl)carbodiimide] and sulfo-NHS ( $N$-hydroxysuccinimide) with a nontoxic level of PEI conjugated to PLGA particles ( $6.5 \mu \mathrm{g}$ of PEI/mg of PLGA), as shown in previous works $(33,51)$. PEI-modified MPs were pelleted by centrifugation for $20 \mathrm{~min}$ at $3000 \mathrm{~g}$, washed with $1 \mathrm{M} \mathrm{NaCl}$ solution two times, and washed with DI water once. PEI-modified NPs were pelleted by ultracentrifugation for $20 \mathrm{~min}$ at $22,000 \mathrm{~g}$, washed with $1 \mathrm{M} \mathrm{NaCl}$ solution two times, and washed with DI water once. Both PEI-modified particle formulations were flash-frozen in liquid nitrogen and lyophilized for 48 hours and stored at $-20^{\circ} \mathrm{C}$. Measurements of MP or NP size and zeta potential were performed in $1 \mathrm{mM} \mathrm{KCl}$ solution with a Malvern Zetasizer. Loading of CpG was quantified using the Nucleic Acid Quantification module of the Gen5 software on a BIOTEK Synergy HT plate reader. MPLA (syn- thetic MPLA-PHAD) encapsulation was measured using LC-MS (52), GC-MS (53), and a surrogate method using fluorometry.

For both LC-MS and GC-MS, MPLA (synthetic MPLA-PHAD) from PLGA particles was extracted by a two-step extraction process, as reported previously (52). Briefly, $2 \mathrm{mg}$ of PLGA particles was first dispersed in $1 \mathrm{ml}$ of acetonitrile, followed by centrifugation at $20,000 \mathrm{~g}$ for $20 \mathrm{~min}$. The supernatant was removed, and the residue was again extracted by adding $500 \mu \mathrm{l}$ of 4:1 chloroform:methanol mixture, followed by centrifugation at $20,000 \mathrm{~g}$ for $20 \mathrm{~min}$.

For LC-MS, dried residues following MPLA extraction from PLGA particles as mentioned above were reconstituted in $1 \mathrm{ml}$ of $4: 1 \mathrm{CHCl}_{3}: \mathrm{IPA}$ (chloroform:isopropyl alcohol) with vortex mixing and sonication. In addition, a dried standard of MPLA was reconstituted in 4:1 $\mathrm{CHCl}_{3}: \mathrm{IPA}$ and was diluted to $0.0625,0.125,0.25,0.5,1,2$, and $5 \mu \mathrm{g}$ $\mathrm{ml}^{-1}$ in a blank sample matrix. A second $1 \mathrm{ml}$ of extraction solvent was added to each sample with vortex mixing. Insoluble material in all samples and standards was removed with centrifugation before transferring $50 \mu \mathrm{l}$ of supernatant to polypropylene LC vial. Residual MPLA on the injection needle required three injections of extraction solvent between each standard or sample injection. Ultraperformance LC-MS (UPLC-MS) was performed using Vanquish (Thermo Fisher Scientific), fitted with a Waters Corporation ACQUITY UPLC BEH C 8 column $(2.1 \times 100 \mathrm{~mm}, 1.7 \mu \mathrm{m}$ particle size), and coupled to a high-resolution accurate mass Orbitrap ID-X Tribrid mass spectrometer system (Thermo Fisher Scientific). The chromatographic method for sample analysis involved elution with 40:60 water:acetonitrile with $10 \mathrm{mM}$ ammonium formate and $0.1 \%$ formic acid (mobile phase A) and 10:90 acetonitrile:IPA with $10 \mathrm{mM}$ ammonium formate and $0.1 \%$ formic acid (mobile phase B) using the following gradient program: $0 \mathrm{~min} 60 \% \mathrm{~A}, 0.1 \mathrm{~min} 60 \% \mathrm{~A}$, 1.5 min $15 \%$ A, 2.8 min $8 \%$ A, 2.9 min $0 \%$ A, held to 4.25 min $0 \%$ A, $4.35 \mathrm{~min} 60 \% \mathrm{~A}$, and held until $5.3 \mathrm{~min}$. The flow rate was set at $0.40 \mathrm{ml} \mathrm{min}{ }^{-1}$. The column temperature was set to $50^{\circ} \mathrm{C}$, and the injection volume was $1 \mu \mathrm{l}$.

The Orbitrap ID-X is a tribrid spectrometer that uses quadrupole isolation with dual detectors, an orbitrap and an ion trap, with a maximum resolving power of 500,000 FWHM (full width at half maximum) at mass/charge ratio $(\mathrm{m} / \mathrm{z}) 200$ and mass accuracy of $<1 \mathrm{ppm}$ (parts per million). The heated electrospray ionization (HESI) source was operated at a vaporizer temperature of $275^{\circ} \mathrm{C}$, a spray voltage of $3.5 \mathrm{kV}$, and sheath, auxiliary, and sweep gas flows of 40 , 8 , and 1 , respectively. The instrument acquired full MS data in the 150 to $2000 \mathrm{~m} / z$ range in positive ionization mode at a resolution of 30,000 in the orbitrap. Tandem MS (MS/MS) experiments were acquired mass spectra using a parallel reaction monitoring method and collected in the ion trap. MS/MS experiments targeted $1763.3 \mathrm{~m} / \mathrm{z}$ with an isolation window of $0.8 \mathrm{~m} / z$ and activated the precursor with collision-induced dissociation at $45 \%$ relative intensity. Before the analysis, the instrument was calibrated with Pierce LTQ Velos ESI positive ion calibration solution (Thermo Fisher Scientific). Data acquisition and processing was carried out using Thermo Scientific Xcalibur (Thermo Fisher Scientific, version 4.3.73.11). The target peak within the range of 1727.8 to $1728.5 \mathrm{~m} / z$ was extracted and integrated for analysis. A five-point calibration curve was collected and used to quantitate the GLA (glycopyranoside Lipid A) in the samples.

For GC-MS, dried residues following extraction of MPLA from PLGA particle were trans-esterified and analyzed, as reported previously (53). In addition, all MPLA standards (100 to $0 \mu \mathrm{g} / \mathrm{ml})$ were also trans-esterified using the same method. Each dried trans-esterified 
sample was dissolved in $400 \mu \mathrm{l}$ of hexane, of which a $100-\mu \mathrm{l}$ aliquot was transferred to a glass insert GC-MS vial for analysis. The sample was analyzed using a HP-5MS column $(30 \mathrm{~m} \times 250 \mu \mathrm{m} \times 0.25 \mu \mathrm{m}$; Agilent J\&W) on an Agilent 7890B GC system equipped with a 5977A mass selective detector and an Agilent 7693 autosampler. MassHunter GC-MS Acquisition software (B.07.00.1413) controlled the instrument. Before the analysis, the instrument was tuned and calibrated with perfluorotributylamine. Samples were injected at $1-\mu$ l volumes and separated with the GC gradient that initiated at $50^{\circ} \mathrm{C}$, ramped at $10^{\circ} \mathrm{C} \mathrm{min}^{-1}$ until $300^{\circ} \mathrm{C}$, and then held for $4 \mathrm{~min}$. The carrier gas helium was at a constant flow rate of $1.2 \mathrm{ml} \mathrm{min}^{-1}$. The inlet was set to $250^{\circ} \mathrm{C}$, split of 20:1, and a split flow of $28.2 \mathrm{ml} \mathrm{min}^{-1}$. The mass spectrometer transfer line, source, and quadrupole were set to $280^{\circ}, 230^{\circ}$, and $150^{\circ} \mathrm{C}$, respectively. The data collection was in full-scan mode with a range of 50 to $500 \mathrm{~m} / z$ at a frequency of 1.7 scans per second. MassHunter software was used to detect and to integrate peaks. A five-point calibration curve was collected with samples after transesterification to quantitate levels of fatty acid methyl esters from MPLA.

For surrogate fluorometry method of assessing MPLA encapsulation, fluorescent dye-conjugated parent lipid, LPS (FITC-LPS, Sigma-Aldrich, catalog no. F8666) was encapsulated in PLGA particles (MPs and NPs) using a double-emulsion method. Then, freezedried FITC-LPS-encapsulated PLGA particles ( $5 \mathrm{mg}$ ) were dissolved in $2 \mathrm{ml}$ of $0.1 \mathrm{M} \mathrm{NaOH}$ solution and incubated overnight to solubilize particle. The next day, FITC-LPS in the solution was analyzed using a fluorometer. Encapsulation efficiency of FITC-LPS in the PLGA particles was determined by calculating the percentage of FITCLPS quantity measured in the PLGA particle to the initial quantity of FITC-LPS used for the preparation of particles.

\section{In vitro activation of mouse BM-APCs with PLP adjuvant formulations}

On day 7 of culture, BM-APCs were plated at a density of 300,000 cells per well in 96-well plates and allowed to settle for 2 hours before the addition of PLP adjuvants. Cell density-to-particle ratio was preserved and extrapolated to encompass six-well plates and 10$\mathrm{cm}$ petri dishes for larger experiments. After treatment with PLP adjuvants, supernatants were harvested at $0.5,1,2,4,6,12$, and 24 hours. ELISA (enzyme-linked immunosorbent assay) or Luminex (Bio-Techne, Minneapolis, MN) was used to measure cytokine concentrations after cell activation (IFN- $\beta$, IL-12p70, IL-6, TNF- $\alpha$, and IL-10). BM-APCs were lysed using Cell Lysis Buffer (Cell Signaling Technology, Danvers, MA; catalog no. 9803S) supplemented with phenylmethylsulfonyl fluoride (PMSF) (Cell Signaling Technology, Danvers, MA; catalog no. 8553S) to collect proteins for Western blots. For Phos-tag gels, BM-APCs were lysed using an EDTA-free lysis buffer of $50 \mathrm{mM}$ tris- $\mathrm{HCl}$ (pH 7.4), $150 \mathrm{mM} \mathrm{NaCl}, 1 \% \mathrm{NP}-40$, $0.5 \%$ sodium deoxycholate, $0.1 \%$ SDS supplemented with complete protease inhibitor (Roche, catalog no. 11836170001), and PhosSTOP phosphatase inhibitor cocktail tablets (Roche, catalog no. 04906837001) (54). Cell fractionation kit (Cell Signaling Technology, Danvers, MA; catalog no. 9038S) supplemented with PMSF and protease inhibitor cocktail (Cell Signaling Technology, Danvers, MA; catalog nos. 8553S and 5871S) was used to separate nuclear from cytoplasmic proteins. Directions were followed as provided by the manufacturer.

\section{Intracellular cytokine response by flow cytometry}

BM-APCs were plated at 1.5 million cells $/ \mathrm{ml}$ in Gibco RPMI 1640 medium (Thermo Fisher Scientific, Waltham, MA) supplemented with
10\% FBS (HyClone, Logan, UT), 1\% penicillin/streptomycin (HyClone, Logan, UT), $1 \mathrm{mM}$ sodium pyruvate, $55 \mu \mathrm{M}$ 2-mercaptoethanol, and GM-CSF (20 ng/ml; PeproTech, Rocky Hill, NJ) with 300,000 cells per well in a 96-well plate and treated with PLPs. Brefeldin A was added to cells at 2 hours to enhance intracellular cytokine accumulation, and cells were harvested at 6 hours for intracellular staining. BM-APCs were stained for live/dead discrimination with Zombie Green Fixable Viability Kit (BioLegend, San Diego, CA) and blocked with anti-mouse CD16/CD32 (clone 93) and True-Stain Monocyte Blocker (BioLegend, San Diego, CA). For surface staining, BMDCs were incubated with BUV395 anti-CD11b (M1/70, BD), BV421 anti-CD11c (N418, BioLegend, San Diego, CA), phycoerythrin (PE)Dazzle 594 anti-Ly6C (HK1.4, BioLegend, San Diego, CA), and allophycocyanin-Cy7 anti-(I-A/I-E) (M5/114.15.2, BioLegend, San Diego, CA). For intracellular staining, cells were fixed with BD Cytofix and permeabilized with Perm/Wash Buffer (BD) and then labeled with allophycocyanin anti-IL-12(p40/p70) (C15.6, BD) or PE anti-IL-6 (MP5-20F3, BioLegend, San Diego, CA) in combination with allophycocyanin anti-TNFa (MP6-XT22, BioLegend, San Diego, CA).

\section{PLP uptake by flow cytometry}

BM-APCs were cultured with PLPs using the same protocol above, except PLGA NP and MP were labeled with customized IR700-CpG ODN 1826 (IDT) to detect cell uptake of PLPs with flow cytometry. Cells were harvested at 24 hours and stained for live/dead discrimination with Zombie Green Fixable Viability Kit (BioLegend, San Diego, CA) and blocked with anti-mouse CD16/CD32 (clone 93) and True-Stain Monocyte Blocker (BioLegend, San Diego, CA). For surface staining, BMDCs were incubated with BUV395 anti-CD11b (M1/70, BD), BV421 anti-CD11c (N418, BioLegend, San Diego, CA), PE-Dazzle 594 anti-Ly6C (HK1.4, BioLegend, San Diego, CA), and $\mathrm{PE}$ anti-(I-A/I-E) (M5/114.15.2, Invitrogen).

\section{Western blots}

Mouse BM-APCs were lysed in $1 \times$ Cell Lysis Buffer (Cell Signaling Technology, catalog no. 9803), EDTA-free lysis buffer (54), or Cell Fractionation Kit (Cell Signaling Technology) according to Western blot, SDS-polyacrylamide gel electrophoresis (PAGE), Phos-tag, or nuclear fractionation. Appropriate volume of lamellae buffer was added to the lysate $(2 \times$ for SDS-PAGE and Phos-tag samples and $6 \times$ for cell fractionation samples). Samples were mechanically homogenized by the French press method using 28-gauge insulin syringe before boiling at $96^{\circ} \mathrm{C}$ for $10 \mathrm{~min}$ and cooled on ice. Equal lysate concentrations were then loaded onto a Bio-Rad mini PROTEAN TGX gel or $50 \mu \mathrm{M}$ Phos-tag acrylamide gel and electrophoresis on either Bio-Rad gel tank or Life Technologies Mini gel tank, respectively. After gel electrophoresis, gel was transferred by either Bio-Rad Trans-Blot turbo or wet tank transfer at room temperature onto a polyvinylidene difluoride (PVDF) membrane following the protocol provided for each gel type (SDS-PAGE or Phos-tag). Transferred PVDF was blocked with 5\% Bio-Rad blocking buffer milk (catalog no. 170-6404) in tris-buffered saline and Tween 20 for 3 hours, rocking at $4^{\circ} \mathrm{C}$. Then, subsequent primary antibody incubation was done overnight at $4^{\circ} \mathrm{C}$ (all primary antibodies were diluted in $1 \%$ blocking milk or for Phos-tag with MBL Max blot solution 1 or Takara Western blot immune booster). Proteins of interest were detected with anti-rabbit IgG (immunoglobulin G) HRP (horseradish peroxidase) (Cell Signaling Technology) and visualized with Bio-Rad Clarity Max according to the manufacturer's protocol. 
Antibodies purchased from Abcam were as follows: IRF5 Chip grade (1:3000; catalog no. ab21689); Cell Signaling Technology: IRF5 (1:1000; catalog no. 4950S) and IRF3 (1:1000; catalog no. 4302S); and Thermo Fisher Scientific/Invitrogen: phosphorylated IRF3 (Ser ${ }^{396}$ ) (1:1000; catalog no. MA5-14947) and TRAF6 rabbit monoclonal (1:1000; catalog no. 702286). Secondary antibodies were from Cell Signaling Technology: anti-rabbit HRP labeled (1:10,000; catalog no. $7074 \mathrm{P}$ ).

Loading controls were visualized by either Gapdh (GA1R) mouse monoclonal (1:5000; Invitrogen, catalog no. MA5-15738), monoclonal $\beta$-actin (1:5000; Sigma-Aldrich, catalog no. A5316), rabbit monoclonal of vimentin (D21H3) (1:1000; Cell Signaling Technology, catalog no. 5741), histone (D1H3) (1:1000; Cell Signaling Technology, catalog no. 4499), or vinculin (1:1000; Cell Signaling Technology, cata$\log$ no. 4650).

\section{Statistical analysis}

All statistical analyses were performed using GraphPad Prism 8. Data are presented in bar graphs as means \pm SD. To assess the statistical significance of the difference between three or more normal datasets, a one-way analysis of variance (ANOVA) was performed. Multiple comparisons were evaluated using Tukey's test, and $P$ values less than 0.05 were considered significant between two groups. GraphPad Prism 8 and JMP Pro 15 were used to create graphs, and BioRender was used to create illustrations.

\section{SUPPLEMENTARY MATERIALS}

Supplementary material for this article is available at http://advances.sciencemag.org/cgi/ content/full/7/3/eabd4235/DC1

\section{REFERENCES AND NOTES}

1. S. G. Reed, M. T. Orr, C. B. Fox, Key roles of adjuvants in modern vaccines. Nat. Med. 19, 1597-1608 (2013).

2. T. W. Dubensky Jr., S. G. Reed, Adjuvants for cancer vaccines. Semin. Immunol. 22, 155-161 (2010).

3. R. K. Gherardi, J. Aouizerate, J. Cadusseau, S. Yara, F. J. Authier, Aluminum adjuvants of vaccines injected into the muscle: Normal fate, pathology and associated disease. Morphologie 100, 85-94 (2016).

4. T. H. Mogensen, Pathogen recognition and inflammatory signaling in innate immune defenses. Clin. Microbiol. Rev. 22, 240-273 (2009).

5. T. Querec, S. Bennouna, S. Alkan, Y. Laouar, K. Gorden, R. Flavell, S. Akira, R. Ahmed, B. Pulendran, Yellow fever vaccine YF-17D activates multiple dendritic cell subsets via TLR2, 7, 8, and 9 to stimulate polyvalent immunity. J. Exp. Med. 203, 413-424 (2006).

6. F. Steinhagen, T. Kinjo, C. Bode, D. M. Klinman, TLR-based immune adjuvants. Vaccine $\mathbf{2 9}$, 3341-3355 (2011).

7. H. Y. Yong, D. Luo, RIG-I-like receptors as novel targets for pan-antivirals and vaccine adjuvants against emerging and re-emerging viral infections. Front. Immunol. 9, 1379 (2018).

8. M. Motwani, S. Pesiridis, K. A. Fitzgerald, DNA sensing by the cGAS-STING pathway in health and disease. Nat. Rev. Genet. 20, 657-674 (2019).

9. H. Xie, I. Gursel, B. E. Ivins, M. Singh, D. T. O'Hagan, J. B. Ulmer, D. M. Klinman, CpG oligodeoxynucleotides adsorbed onto polylactide-co-glycolide microparticles improve the immunogenicity and protective activity of the licensed anthrax vaccine. Infect. Immun. 73, 828-833 (2005).

10. B. Sun, T. Xia, Nanomaterial-based vaccine adjuvants. J. Mater. Chem. B 4, 5496-5509 (2016).

11. T. J. Moyer, A. C. Zmolek, D. J. Irvine, Beyond antigens and adjuvants: Formulating future vaccines. J. Clin. Invest. 126, 799-808 (2016).

12. J. Wu, G. Ma, Imitation of nature: Bionic design in the study of particle adjuvants. J. Control. Release 303, 101-108 (2019).

13. S. L. Demento, A. L. Siefert, A. Bandyopadhyay, F. A. Sharp, T. M. Fahmy, Pathogenassociated molecular patterns on biomaterials: A paradigm for engineering new vaccines. Trends Biotechnol. 29, 294-306 (2011).

14. H. Liu, K. D. Moynihan, Y. Zheng, G. L. Szeto, A. V. Li, B. Huang, D. S. van Egeren, C. Park, D. J. Irvine, Structure-based programming of lymph-node targeting in molecular vaccines. Nature 507, 519-522 (2014).
15. D. J. Irvine, M. A. Swartz, G. L. Szeto, Engineering synthetic vaccines using cues from natural immunity. Nat. Mater. 12, 978-990 (2013).

16. R. Toy, K. Roy, Engineering nanoparticles to overcome barriers to immunotherapy. Bioeng. Transl. Med. 1, 47-62 (2016).

17. B. Kwong, H. Liu, D. J. Irvine, Induction of potent anti-tumor responses while eliminating systemic side effects via liposome-anchored combinatorial immunotherapy. Biomaterials 32, 5134-5147 (2011).

18. M. C. Hanson, M. P. Crespo, W. Abraham, K. D. Moynihan, G. L. Szeto, S. H. Chen, M. B. Melo, S. Mueller, D. J. Irvine, Nanoparticulate STING agonists are potent lymph node-targeted vaccine adjuvants. J. Clin. Invest. 125, 2532-2546 (2015).

19. P. Pradhan, H. Qin, J. A. Leleux, D. Gwak, I. Sakamaki, L. W. Kwak, K. Roy, The effect of combined IL10 siRNA and CpG ODN as pathogen-mimicking microparticles on Th1/ Th2 cytokine balance in dendritic cells and protective immunity against B cell lymphoma. Biomaterials 35, 5491-5504 (2014).

20. E.-J. Ko, Y. Lee, Y.-T. Lee, Y.-J. Kim, K.-H. Kim, S.-M. Kang, MPL and CpG combination adjuvants promote homologous and heterosubtypic cross protection of inactivated split influenza virus vaccine. Antiviral Res. 156, 107-115 (2018).

21. R. Kuai, X. Sun, W. Yuan, L. J. Ochyl, Y. Xu, A. Hassani Najafabadi, L. Scheetz, M.-Z. Yu, I. Balwani, A. Schwendeman, J. J. Moon, Dual TLR agonist nanodiscs as a strong adjuvant system for vaccines and immunotherapy. J. Control. Release 282, 131-139 (2018).

22. P. U. Atukorale, S. P. Raghunathan, V. Raguveer, T. J. Moon, C. Zheng, P. A. Bielecki, M. L. Wiese, A. L. Goldberg, G. Covarrubias, C. J. Hoimes, E. Karathanasis, Nanoparticle encapsulation of synergistic immune agonists enables systemic codelivery to tumor sites and IFN $\beta$-driven antitumor immunity. Cancer Res. 79, 5394-5406 (2019).

23. G. Napolitani, A. Rinaldi, F. Bertoni, F. Sallusto, A. Lanzavecchia, Selected Toll-like receptor agonist combinations synergistically trigger a $T$ helper type 1-polarizing program in dendritic cells. Nat. Immunol. 6, 769-776 (2005).

24. T. K. Ghosh, D. J. Mickelson, J. C. Solberg, K. E. Lipson, J. R. Inglefield, S. S. Alkan, TLR-TLR cross talk in human PBMC resulting in synergistic and antagonistic regulation of type-1 and 2 interferons, IL-12 and TNF- $\alpha$. Int. Immunopharmacol. 7, 1111-1121 (2007).

25. K. K. Short, S. M. Miller, L. Walsh, V. Cybulski, H. Bazin, J. T. Evans, D. Burkhart, Coencapsulation of synthetic lipidated TLR4 and TLR7/8 agonists in the liposomal bilayer results in a rapid, synergistic enhancement of vaccine-mediated humoral immunity. J. Control. Release 315, 186-196 (2019).

26. S. P. Kasturi, I. Skountzou, R. A. Albrecht, D. Koutsonanos, T. Hua, H. I. Nakaya, R. Ravindran, S. Stewart, M. Alam, M. Kwissa, F. Villinger, N. Murthy, J. Steel, J. Jacob, R. J. Hogan, A. García-Sastre, R. Compans, B. Pulendran, Programming the magnitude and persistence of antibody responses with innate immunity. Nature 470, 543-547 (2011)

27. H. An, Y. Yu, M. Zhang, H. Xu, R. Qi, X. Yan, S. Liu, W. Wang, Z. Guo, J. Guo, Z. Qin, X. Cao, Involvement of ERK, p38 and NF- $\mathrm{KB}$ signal transduction in regulation of TLR2, TLR4 and TLR9 gene expression induced by lipopolysaccharide in mouse dendritic cells. Immunology 106, 38-45 (2002).

28. N. N. Kuzmich, K. V. Sivak, V. N. Chubarev, Y. B. Porozov, T. N. Savateeva-Lyubimova, F. Peri, TLR4 signaling pathway modulators as potential therapeutics in inflammation and sepsis. Vaccines (Basel) 5, 34 (2017).

29. M. D. Halpern, R. J. Kurlander, D. S. Pisetsky, Bacterial DNA induces murine interferon- $\gamma$ production by stimulation of interleukin- 12 and tumor necrosis factor- $\alpha$. Cell. Immunol. 167, 72-78 (1996).

30. D. M. Klinman, A. K. Yi, S. L. Beaucage, J. Conover, A. M. Krieg, CpG motifs present in bacteria DNA rapidly induce lymphocytes to secrete interleukin 6, interleukin 12 , and interferon gamma. Proc. Natl. Acad. Sci. U.S.A. 93, 2879-2883 (1996),

31. R. Madan-Lala, P. Pradhan, K. Roy, Combinatorial delivery of dual and triple TLR agonists via polymeric pathogen-like particles synergistically enhances innate and adaptive immune responses. Sci. Rep. 7, 2530 (2017).

32. Z. Yang, L. Wang, H. Yu, R. Wang, Y. Gou, M. M. Zhang, C. Kang, T. Liu, Y. Lan, X. Wang, J. Liu, M. A. Cooper, X. Li, K. Yue, Y. Yu, L. Wang, B. Y. S. Kim, W. Jiang, W. Sun, Membrane TLR9 positive neutrophil mediated MPLA protects against fatal bacterial sepsis. Theranostics 9, 6269-6283 (2019).

33. J. A. Leleux, P. Pradhan, K. Roy, Biophysical attributes of $\mathrm{CpG}$ presentation control TLR 9 signaling to differentially polarize systemic immune responses. Cell Rep. 18, 700-710 (2017).

34. P. Pradhan, J. Leleux, J. Liu, K. Roy, A simple, clinically relevant therapeutic vaccine shows long-term protection in an aggressive, delayed-treatment B lymphoma model. JCI Insight 2, e92522 (2017)

35. J. Helft, J. Böttcher, P. Chakravarty, S. Zelenay, J. Huotari, B. U. Schraml, D. Goubau, C. Reis e Sousa, GM-CSF mouse bone marrow cultures comprise a heterogeneous population of $\mathrm{CD} 11 \mathrm{c}^{+} \mathrm{MHCI}{ }^{+}$macrophages and dendritic cells. Immunity 42, 1197-1211 (2015).

36. Z. Erlich, I. Shlomovitz, L. Edry-Botzer, H. Cohen, D. Frank, H. Wang, A. M. Lew, K. E. Lawlor, Y. Zhan, J. E. Vince, M. Gerlic, Macrophages, rather than DCs, are responsible for inflammasome activity in the GM-CSF BMDC model. Nat. Immunol. 20, 397-406 (2019). 
37. Y. R. Na, D. Jung, G. J. Gu, S. H. Seok, GM-CSF grown bone marrow derived cells are composed of phenotypically different dendritic cells and macrophages. Mol. Cells 39, 734-741 (2016).

38. W. E. Purtha, M. Swiecki, M. Colonna, M. S. Diamond, D. Bhattacharya, Spontaneous mutation of the Dock2 gene in $/ r f 5^{-/-}$mice complicates interpretation of type I interferon production and antibody responses. Proc. Natl. Acad. Sci. U.S.A. 109, E898-E904 (2012).

39. M. Zhu, X. Ding, R. Zhao, X. Liu, H. Shen, C. Cai, M. Ferrari, H. Y. Wang, R.-F. Wang, Co-delivery of tumor antigen and dual toll-like receptor ligands into dendritic cell by silicon microparticle enables efficient immunotherapy against melanoma. J. Control. Release 272, 72-82 (2018)

40. N. D. Pennock, L. Gapin, R. M. Kedl, IL-27 is required for shaping the magnitude, affinity distribution, and memory of T cells responding to subunit immunization. Proc. Natl. Acad. Sci. U.S.A. 111, 16472-16477 (2014).

41. Y. Song, J. Nigro, L. Yu, M. Congiu, N. Skinner, A. Thompson, K. Visvanathan, Secreted and intracellular cytokines are complementary measures for human monocytes treated with Toll-like receptor agonists. J. Immunol. Methods 464, 131-137 (2019).

42. S. L. Latty, J. Sakai, L. Hopkins, B. Verstak, T. Paramo, N. A. Berglund, E. Cammarota, P. Cicuta, N. J. Gay, P. J. Bond, D. Klenerman, C. E. Bryant, Activation of Toll-like receptors nucleates assembly of the MyDDosome signaling hub. eLife 7, e31377 (2018).

43. H. Shen, B. M. Tesar, W. E. Walker, D. R. Goldstein, Dual signaling of MyD88 and TRIF is critical for maximal TLR4-induced dendritic cell maturation. J. Immunol. 181, 1849-1858 (2008).

44. G. N. Zhao, D. S. Jiang, H. Li, Interferon regulatory factors: At the crossroads of immunity, metabolism, and disease. Biochim. Biophys. Acta 1852, 365-378 (2015).

45. J. Zhang, D. Chang, Y. Yang, X. Zhang, W. Tao, L. Jiang, X. Liang, H. Tsai, L. Huang, L. Mei, Systematic investigation on the intracellular trafficking network of polymeric nanoparticles. Nanoscale 9, 3269-3282 (2017).

46. H. Huang, B. Beautler, CpG11 is an allele of Tlr9 and causes Tlr9 signaling defects. MGI Direct Data Submission (2010).

47. H. Negishi, H. Yanai, A. Nakajima, R. Koshiba, K. Atarashi, A. Matsuda, K. Matsuki, S. Miki, T. Doi, A. Aderem, J. Nishio, S. T. Smale, K. Honda, T. Taniguchi, Cross-interference of RLR and TLR signaling pathways modulates antibacterial T cell responses. Nat. Immunol. 13, 659-666 (2012).

48. A. Takaoka, H. Yanai, S. Kondo, G. Duncan, H. Negishi, T. Mizutani, S. I. Kano, K. Honda, Y. Ohba, T. W. Mak, T. Taniguchi, Integral role of IRF-5 in the gene induction programme activated by Toll-like receptors. Nature 434, 243-249 (2005).

49. M. Hedl, J. Yan, C. Abraham, IRF5 and IRF5 disease-risk variants increase glycolysis and human M1 macrophage polarization by regulating proximal signaling and Akt2 activation. Cell Rep. 16, 2442-2455 (2016).

50. X. Ouyang, H. Negishi, R. Takeda, Y. Fujita, T. Taniguchi, K. Honda, Cooperation between MyD88 and TRIF pathways in TLR synergy via IRF5 activation. Biochem. Biophys. Res. Commun. 354, 1045-1051 (2007)
51. S. P. Kasturi, K. Sachaphibulkij, K. Roy, Covalent conjugation of polyethyleneimine on biodegradable microparticles for delivery of plasmid DNA vaccines. Biomaterials 26 6375-6385 (2005).

52. S. Hamdy, A. Haddadi, V. Somayaji, D. Ruan, J. Samuel, Pharmaceutical analysis of synthetic lipid A-based vaccine adjuvants in poly (D,L-lactic-co-glycolic acid) nanoparticle formulations. J. Pharm. Biomed. Anal. 44, 914-923 (2007).

53. M. A. Masood, J. Blonder, T. D. Veenstra, Quantitation of monophosphorylated lipid $A$ in the oil-in-water adjuvant delivery systems using transesterification and GC-MS. J. Pharm. Sci. 106, 1760-1763 (2017).

54. G. R. Sato, T. Ban, T. Tamura, Phos-tag immunoblot analysis for detecting IRF5 phosphorylation. Bio-Protocol 7, e2295 (2017).

Acknowledgments: We acknowledge the assistance of C. Young and J. Yao in the maintenance of the breeding colony for KO mice used in these studies, M. C. Keenum for assistance with particle synthesis and characterization, and N. Narang and G. Vogel for technical assistance with studies evaluating immune response kinetics. Funding: We acknowledge funding support from NIH grant U01-AI124270-02 and the Robert A. Milton Chaired Professorship to K.R. E.L.B. was supported by the National Science Foundation Graduate Research Fellowship Program (grant no. DGE-1650044). We also acknowledge funding support from the NIH/NIGMS-sponsored Cell and Tissue Engineering (CTEng) Biotechnology Training Program (T32GM008433) to A.B. This work was supported by the Georgia Institute of Technology's Systems Mass Spectrometry Core Facility. Author contributions: P.P., R.T., and K.R. conceptualized studies. P.P., R.T., N.J., A.A., B.P., A.B., and E.L.B. performed the experiments. S.G.M. and D.A.G. developed LC-MS and GC-MS methods for MPLA quantification. P.P., R.T., N.J., and K.R. wrote the manuscript. P.J.S., D.M.S., and K.R. provided resources and guidance and designed experiments. Competing interests: $K . R$. is an inventor on an issued U.S. patent related to this work filed by the University of Texas System (U.S. patent no. 8,399,025, filed on 6 June 2005, issued on 19 March 2013). The authors declare no other competing interests. Data and materials availability: All data needed to evaluate the conclusions in the paper are present in the paper and/or the Supplementary Materials. Additional data related to this paper may be requested from the authors.

Submitted 19 June 2020

Accepted 18 November 2020

Published 13 January 2021

$10.1126 /$ sciadv.abd4235

Citation: P. Pradhan, R. Toy, N. Jhita, A. Atalis, B. Pandey, A. Beach, E. L. Blanchard, S. G. Moore, D. A. Gaul, P. J. Santangelo, D. M. Shayakhmetov, K. Roy, TRAF6-IRF5 kinetics, TRIF, and biophysical factors drive synergistic innate responses to particle-mediated MPLA-CpG co-presentation. Sci. Adv. 7, eabd4235 (2021). 